\title{
The somatic marker hypothesis: A neural theory of economic decision
}

\author{
Antoine Bechara*, Antonio R. Damasio \\ Department of Neurology, University of Iowa, 200 Hawkins Drive, Iowa City, IA 52242, USA
}

Received 8 June 2004

Available online 23 September 2004

\begin{abstract}
Modern economic theory ignores the influence of emotions on decision-making. Emerging neuroscience evidence suggests that sound and rational decision making, in fact, depends on prior accurate emotional processing. The somatic marker hypothesis provides a systems-level neuroanatomical and cognitive framework for decision-making and its influence by emotion. The key idea of this hypothesis is that decision-making is a process that is influenced by marker signals that arise in bioregulatory processes, including those that express themselves in emotions and feelings. This influence can occur at multiple levels of operation, some of which occur consciously, and some of which occur non-consciously. Here we review studies that confirm various predictions from the hypothesis, and propose a neural model for economic decision, in which emotions are a major factor in the interaction between environmental conditions and human decision processes, with these emotional systems providing valuable implicit or explicit knowledge for making fast and advantageous decisions. (c) 2004 Elsevier Inc. All rights reserved.
\end{abstract}

\section{Introduction}

Modern economic theory assumes that human decision-making involves rational Bayesian maximization of expected utility, as if humans were equipped with unlimited knowledge, time, and information-processing power. The influence of emotions on

\footnotetext{
* Corresponding author.

E-mail address: antoine-bachara@uiowa.edu (A. Bechara).
} 
decision-making is largely ignored. Indeed, the development of what became known as the "Expected Utility" theory was really based on the idea that people established their values for wealth on the basis of the pain and pleasure that it would give them. So "Utility" was conceived as a balance of pleasure and pain. These notions of pleasure and pain were eliminated from notions of utility in subsequent economic models. The exclusion of current economic models of expected utility to the role of emotion in human decisions is therefore inconsistent with their foundations.

Perhaps economists have ignored the role of emotions in decision-making because emotions had a checkered history in psychology and neuroscience; there was disagreement on how to define them, disagreement on what they are for, and what to include them in. Furthermore, according to a popular notion that most of us learn early in life, rational calculation forms the basis of sound decisions; "emotion has no IQ" and can only interfere with good judgment. Could it be, however, that these notions are wrong and that emotion plays a role in sound, rational decision making? That is precisely what studies of decision-making in neurological patients with impaired emotion processing suggest. These studies have been the basis for the somatic marker hypothesis, and the aim of this article is to use that hypothesis, a systems-level cognitive and neuroanatomical framework for decision-making, to address the problem of economic decisions. In our view, the two fields of economics and neuroscience have much to learn from one another, especially in the area of decision-making, and that the time has come for direct, explicit communication between the two disciplines. Thus guided by this framework, we argue that

(1) knowledge and reasoning alone are usually not sufficient for making advantageous decisions, and that the role of emotion in decision-making has been underestimated;

(2) that emotion is beneficial to decision-making when it is integral to the task, but can be disruptive when it is unrelated to the task; and

(3) that the implementation of decisions under certainty or uncertainty engage different neural circuitry.

\section{An overview of neurological investigations of decision-making}

In the past 15 years, we studied several patients with lesions of the ventromedial prefrontal (VM) cortex who showed impairments in judgment and decision-making in real-life settings, in spite of maintaining a normal intellect (Fig. 1). The case of Phineas Gage paved the way for the notion that the frontal lobes were linked to judgement, decision-making, social conduct, and personality. A number of cases of frontal lobe damage with defects similar to those of Phineas Gage appeared in the literature (e.g., see Ackerly and Benton, 1948; Brickner, 1932; Welt, 1888 for descriptions), but received little attention. A greater interest in the decision-making and social aspects of the "frontal lobe syndrome" was triggered in part by the description of a modern counterpart to Phineas Gage (Eslinger and Damasio, 1985).

Patients with bilateral damage to the VM prefrontal cortex develop severe impairments in personal and social decision-making. They have difficulties planning their workday, as well as difficulties in choosing friends, partners, and activities. The actions they elect to 


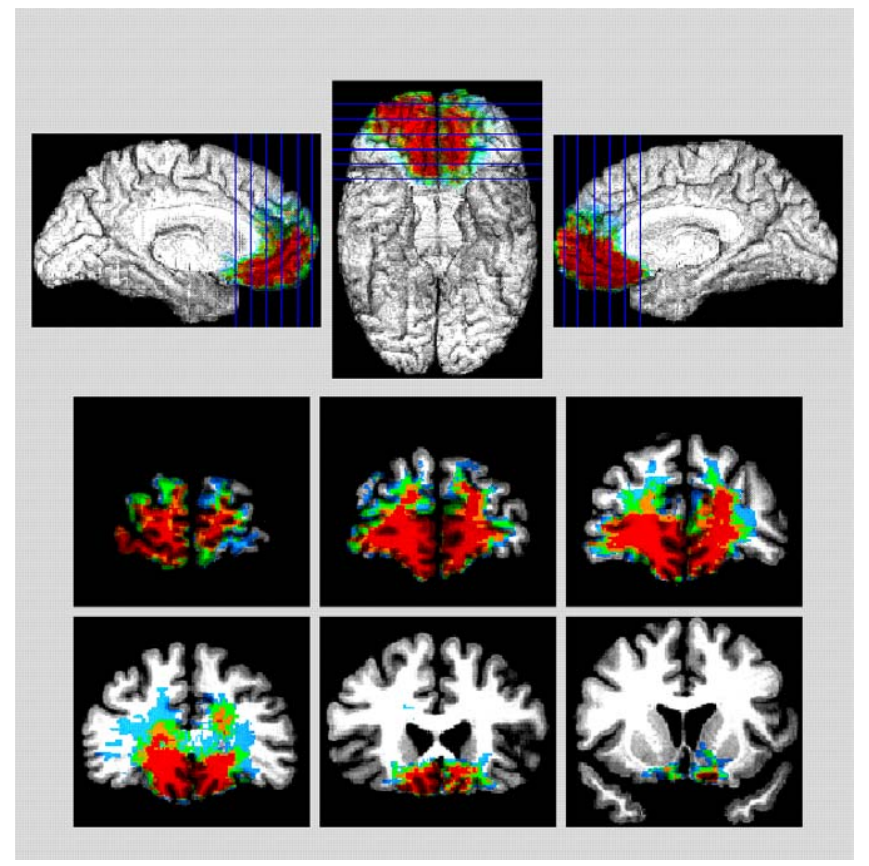

Fig. 1. Overlap of lesions in a group of patients with lesions of the ventromedial prefrontal (VM) cortex. The red color indicates an overlap of 4 or more patients.

pursue often lead to losses of diverse order, e.g., financial losses, losses in social standing, losses of family and friends. The choices they make are no longer advantageous-the patients often decide against their best interests - and are remarkably different from the kinds of choices they were known to make in the pre-morbid period. They are unable to learn from previous mistakes as reflected by repeated engagement in decisions that lead to negative consequences. In striking contrast to this real-life decision-making impairment, problem-solving abilities in laboratory settings remain largely normal. As noted, the patients have normal intellect, as measured by a variety of conventional neuropsychological tests (Bechara et al., 1998; Damasio et al., 1990; Eslinger and Damasio, 1985), a fact that made it difficult to explain these patients' disturbance in terms of defects in knowledge pertinent to the situation, general intellectual compromise, defects in language comprehension or expression, working memory, or attention (Anderson et al., 1999; Anderson et al., 1991; Bechara et al., 1998; Saver and Damasio, 1991). While these VM patients were intact on standard neuropsychological tests, however, they did have a compromised ability to express emotion and experience feelings in appropriate situations, i.e., despite normal intellect, there were abnormalities in emotion and feeling, along with the abnormalities in decision-making. The latter observations led to the somatic marker hypothesis (Damasio, 1994; Damasio et al., 1991). 


\section{The somatic marker hypothesis}

The hypothesis attributes these patients' inability to make advantageous decisions in real-life to a defect in an emotional mechanism that rapidly signals the prospective consequences of an action, and accordingly assists in the selection of an advantageous response option. The hypothesis specifies a number of structures and operations required for the normal operation of decision-making. Deprived of this emotional signal, these patients rely on a reasoned cost-benefit analysis of numerous and often conflicting options involving both immediate and future consequences. The impairment degrades the speed of deliberation (e.g., choosing between two brands of cereal may take a patient a very long time because of endless reasoned analyses of the pros and cons of each brand), and also degrades the adequacy of the choice, i.e., patients may choose disadvantageously. Our studies have focused primarily on the quality, as opposed to the speed, of making decisions.

An emotion is defined as a collection of changes in body and brain states triggered by a dedicated brain system that responds to specific contents of one's perceptions, actual or recalled, relative to a particular object or event (Damasio 1994, 1999, 2003). The specific object or event that predictably causes an emotion is designated as an "emotionally-competent stimulus." The responses toward the body proper enacted in a body (somatic) state involve physiological modifications. These modifications range from changes in internal milieu and viscera that may not be perceptible to an external observer (e.g., endocrine release, heart rate, smooth muscle contraction) to changes in the musculoskeletal system that may be obvious to an external observer (e.g., posture, facial expression, specific behaviors such as freezing, flight and fight, and so on). The responses aimed at the brain lead to

(a) the central nervous system release of certain neurotransmitters (e.g., dopamine, serotonin, acetylcholine, noreadrenaline),

(b) an active modification of the state of somatosensory maps such as those of the insular cortex ("as-if-body-states"), and

(c) to a modification of the transmission of signals from the body to somatosensory regions.

The ensemble of all these enacted responses in the body proper and in the brain constitutes an emotion. The ensemble of signals as mapped in somatosensory regions of the brain itself provide the essential ingredients for what is ultimately perceived as a feeling, a phenomenon perceptible to the individual in whom they are enacted (Damasio, 1999, 2003).

Because the term emotion tends to mean different things to the layman, the psychologist, and the physiologist, we have used the term "somatic" to refer to the collection of bodyrelated responses that hallmark an emotion. Somatic refers to the Greek word "soma," i.e., body.

\subsection{Induction of somatic states}

Somatic states can be induced from

(1) primary inducers, and

(2) secondary inducers (Damasio, 1995). 
Primary inducers are innate or learned stimuli that cause pleasurable or aversive states. Once present in the immediate environment, they automatically and obligatorily elicit a somatic response. Examples of primary inducers include the encounter of a fear object (e.g., a snake), or a stimulus predictive of a fear object. Primary inducers are also concepts or knowledge that through learning can automatically and obligatorily elicit emotional responses, such as hearing that you have won a prize or a lottery ticket, or that your life savings have been lost in a market crash. Humans also automatically, involuntarily, and obligatorily elicit a "pleasure" response when they uncover a solution to a problem. This "aha" reaction to solving a puzzle is also an example of primary inducers.

Secondary inducers, on the other hand, are entities generated by the recall of a personal or hypothetical emotional event, i.e., "thoughts" and "memories" of the primary inducer, which when brought to working memory elicit a somatic state. Examples of secondary inducers include the emotional response elicited by the memory of encountering a snake, or the memory of losing a large sum of money. The imagination of being attacked by a bear, winning an award, or losing a large sum of money, are also examples of secondary inducers.

We see the amygdala as a critical substrate in the neural system necessary for triggering somatic states from primary inducers. By contrast, the ventromedial (VM) prefrontal cortex is a critical substrate in the neural system necessary for triggering somatic states from secondary inducers, although it can be involved in the emotions triggered by some primary inducers as well.

Evidence suggests that, in a normal brain, primary and secondary inducer processing can be elicited by the same stimulus and at the same time. Looking at a picture of a baby with a tumor growth may quickly and automatically trigger an emotional response (serving as a primary inducer), but at the same time, it may generate thoughts (e.g., picturing one's own child in this situation) that operate as a secondary inducer (Bechara et al., 2003). The operations of the primary and secondary inducer systems are difficult to disentangle in a normal brain, and can best be brought to light in patients with lesions in structures critical for the processing of primary or secondary inducers (Bechara et al., 2003).

\subsection{Development of somatic state patterns}

Evidence suggests that the normal development of secondary inducers is contingent upon the normal development of primary inducers, i.e., if the processing of primary inducers were abnormal, then secondary inducer processing would be abnormal too. However, once secondary inducers have been acquired normally, the induction of somatic states by secondary inducers becomes less dependent on primary induction (Bechara et al., 2003). For example, if burning a hand on a hot stove did not induce pain (i.e., primary inducer processing is abnormal), then one will not know how painful it should feel when attempting to touch, for example, boiling water. However, if one used to be able to feel pain and has already developed mental representations of what it feels like to be in pain (i.e., primary induction was normal), then this person is likely to avoid painful situations (i.e., secondary induction can be normal), even after sustaining neurological damage, and becoming un- 
able to feel pain when touching a hot stove. The physiological steps that lead to the normal development of somatic states representations are the following:

(1) The amygdala is an important trigger structure for somatic states from primary inducers. It couples the features of primary inducers, which can be processed subliminally via the thalamus (LeDoux, 1996; Morris et al., 1999) or explicitly via early sensory and high-order association cortices, with the somatic state associated with the inducer. This somatic state is evoked via effector structures such as the hypothalamus and autonomic brainstem nuclei that produce changes in internal milieu and visceral structures along with other effector structures such as the ventral striatum, periacqueductal gray (PAG), and other brainstem nuclei, which produce changes in facial expression and specific approach or withdrawal behaviors. Several lines of animal and human studies support this functional role of the amygdala in triggering somatic states from primary inducers (Bechara et al., 2003).

(2) Once somatic states from primary inducers are induced, signals from these somatic states are relayed to the brain. Signals from activated somatic states lead to the development of somatic state patterns in brainstem nuclei (e.g., the PBN), and in somatosensing cortices (e.g., insular/SII, SI cortices, and cingulate cortices). After a somatic state has been triggered by a primary inducer and experienced at least once, a pattern for this somatic state is formed. The subsequent presentation of a stimulus that evokes thoughts and memories about a specific primary inducer will then operate as a secondary inducer. Secondary inducers are presumed to re-activate the pattern of somatic state belonging to a specific primary inducer and generate a fainter activation of the somatic state than if it were triggered by an actual primary inducer. For example, imagining the loss of a large sum of money (secondary inducer) re-activates the pattern of somatic state belonging to an actual prior experience of money loss (primary inducer). However, the somatic state generated by the imagination of losing a large sun of money is fainter than one triggered by an actual experience of money loss.

(3) Provided that somatic states associated with secondary inducers develop normally, generating somatic states from secondary inducers is dependent on cortical circuitry in which the VM cortex plays a critical role. The VM cortex is a trigger structure for somatic states from secondary inducers. It serves as a convergence-divergence zone, which neuron ensembles can couple

(a) a certain category of event based on memory records in high order association cortices to

(b) the effector structures that execute the somatic state, and to

(c) the neural patterns related to the non-conscious (e.g., in the PBN) or conscious (e.g., in the insula/SII, SI cortices) feeling of the somatic state.

In other words, the VM cortex couples knowledge of secondary inducer events to somatic state patterns related to "what it feels like" to be in a given situation. However, in some instances, the VM cortex couples knowledge of secondary inducer events to covert response effectors at the level of the basal forebrain or brainstem only. The anticipatory SCRs acquired during the pre-hunch period of our experimental gambling task are an example of this instance (Bechara et al., 1997). In this case, consciously pondering on which deck to 
choose from (a secondary inducer) elicits a covert somatic response, which is an expression of the bias process that leads the subject to choose the correct deck without any awareness of why the choice was made. Several lines of studies support the notion just presented, that the VM cortex is a trigger structure for somatic states from secondary inducers (Bechara et al., 2000a; Bechara et al., 2003; Bechara et al., 2002).

\subsection{Somatic state activation during decision-making}

The VM cortices contain convergence-divergence neuron ensembles, which hold a record of temporal conjunctions of activity in varied regions (i.e., sensory cortices and limbic structures) caused by external and internal stimuli. When parts of certain exteroceptiveinteroceptive conjunctions are reprocessed, consciously or non-consciously, their activation is signaled to VM cortices, which in turn activate somatic effectors in hypothalamus, and brainstem nuclei. This latter activity is an attempt to reconstitute the kind of somatic state that belonged to the original conjunction. Two chains of physiologic events are possible at this point (Fig. 2).

\subsubsection{The "body loop" mechanism of somatic markers}

In one chain of physiological events, an appropriate somatic state is actually re-enacted in the body proper, and signals from its activation are then relayed back to subcortical and cortical processing structures, especially in the insular and SII and SI cortices. This anatomical system is described as the "body loop" because it engages the body.

A large number of channels convey body information to the central nervous system (e.g., spinal cord, vagus nerve, humoral signals). Evidence suggests that the vagal route is especially critical (Bechara, 2002), a fact that corroborates previous evidence implicating the vagus nerve in the modulation of memory by emotion (e.g., see Roozendaal et al., 1996 for a review).

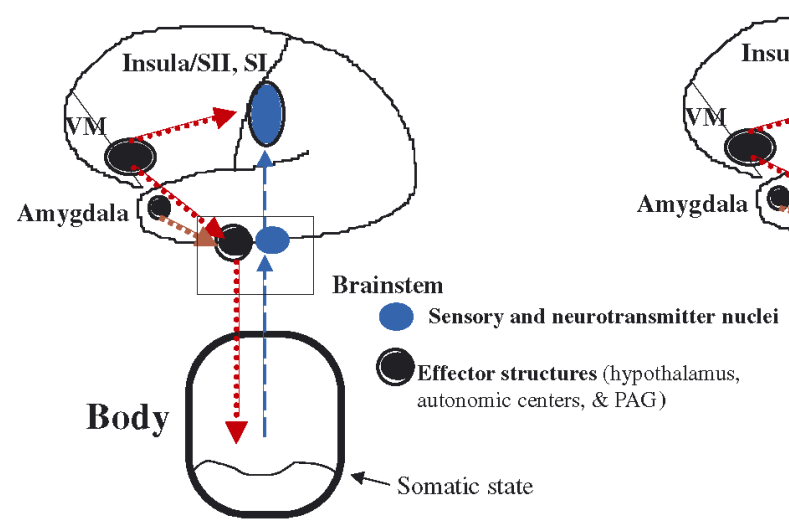

"Body Loop"
"As If Body Loop"

Fig. 2. Simple diagrams illustrating the "body loop" and "as if loop" chain of physiologic events. In both "body loop" and "as if loop" panels, the brain is represented by the top black perimeter and the body by the bottom one. 
The enacted somatic state can then act at conscious or non-conscious level and influence activity in

(1) regions involved in body mapping, i.e., holding patterns of somatic states that help generate feelings;

(2) regions involved in the triggering of somatic states (e.g., amygdala and VM cortex), so that the threshold for triggering subsequent somatic states is increased or decreased;

(3) regions involved in working memory (e.g., dorsolateral prefrontal cortex and other high order association cortices), so that a particular representation is strengthened or weakened.

Indeed, the somatic marker hypothesis has posited that somatic states operate on decisionmaking and working memory (Damasio, 1994). The influence of somatic state signals on the contents displayed in working memory helps endorse or reject "objects" and "response options" (i.e., secondary inducers) brought to mind during the pondering of a decision, i.e., they help bias the options and plans for action; and finally,

(4) somatic state signals influence activity in regions concerned with motor responses and behavioral actions (e.g., striatum and anterior cingulate/supplementary motor area (SMA)).

They interfere with response selection and thus render the occurrence of a given behavior more likely or less likely.

We propose that the biasing action of somatic states on response selection is mediated by the release of neurotransmitters in the telencephalon (i.e., the cerebral cortex) and the diencephalon, which includes the basal ganglia and thalamus. The cell bodies of all major neurotransmitter systems (e.g., dopamine (DA), serotonin (5-HT), noreadrenaline (NA), and acetylcholine (Ach)) are located in the brainstem; the axon terminals of these neurotransmitter neurons synapse on cells and/or terminals all over the telencephalon. When somatic state signals are transmitted to the cell bodies of these neurotransmitter neurons, the signaling influences the pattern of neurotransmitter release at the terminals. In turn, evidence from cellular physiology shows that neurotransmitters modulate synaptic activity by rendering the triggering of action potentials as more likely or less likely (e.g., see Mogenson, 1987 for a review of the modulation effect of DA on striatal neurons). Thus changes in neurotransmitter release induced by somatic state signals modulate the synaptic activities of telencephalic neurons subserving behavior and cognition, thereby providing a mechanism for somatic states to exert a biasing effect on behaviors (e.g., selection of a response over another), feelings, and cognitive patterns.

\subsubsection{The "as if body loop" mechanism of somatic markers}

During the deliberation of decisions, the mental representation of a future event triggers a somatic state, no matter how faint, which may be consciously perceived as a good or bad feeling, or processed unconsciously (Damasio, 1994; Overskeid, 2000). When somatic states from primary or secondary inducers cannot be detected as changes in physiological parameters within the body proper, they can at least be detected as changes in the 
activity of different neurotransmitter systems (Fig. 2). Indeed, the anatomy of these neurotransmitter systems is consistent with this hypothesis, i.e., there are multiple direct and indirect connections between the amygdala and the VM cortex, and the neurotransmitter nuclei within the brainstem (Blessing, 1997; Nauta, 1971). Because after somatic states have been expressed they form patterns in nuclei of the brainstem and insular/SII, SI cortices, one possible chain of physiologic events is to by-pass the body altogether, activate directly the insular/SII, SI cortices, and or the brainstem nuclei holding covert patterns of somatic states. In other words, instead of having somatic states expressed in the body, we propose that the activation of representations of somatic states in the brainstem and/or the cortex can induce changes in neurotransmitter release, without engaging the body. This anatomical system is described as the "as if body loop" because the somatic state is not re-enacted in the body. Although somatic signals are based on structures representing the body and its states, from the brain stem and hypothalamus to the cerebral cortex, the somatic signals do not need to originate in the body in every instance. Somatic states can in fact be "simulated" intra-cerebrally in the "as if body loop."

Thus the neural system mediating the activation of somatic states involves several neural regions:

(a) the VM cortex,

(b) the amygdala,

(c) the somatosensory cortices (insular/SII, SI),

(d) the basal ganglia, anterior cingulate, and brainstem nuclei, and the humeral and neural pathways that signal body states to the central nervous system.

\section{Testing the somatic marker hypothesis}

We have tested the somatic marker hypothesis using the gambling task (GT) paradigm for measuring decision-making (Bechara et al., 2000b). However, there are other paradigms, namely the "gamble" and "risk" tasks developed by Rogers and his colleagues (Rogers et al., 1999a), as well as tasks of delayed discounting (Bickel et al., 1995). It has been shown that there is a significant correlation between performance on the GT, the "gamble task," and tasks of delayed discounting (Monterosso et al., 2001), thus supporting the notion that these three sets of tasks may engage a common mechanism of decisionmaking, tied to the $\mathrm{VM}$ region.

The gambling task (GT) has been described in detail elsewhere (Bechara et al., 2000b). Briefly, in the gambling task, subjects have to choose between decks of cards which yield high immediate gain but larger future loss, i.e., long term loss, and decks which yield lower immediate gain but a smaller future loss, i.e., a long term gain. The task consists of 4 decks of cards named $A, B, C$, and $D$. The goal in the task is to maximize profit on a loan of play money. Subjects are required to make a series of 100 card selections. However, they are not told ahead of time how many card selections they are going to make. Subjects can select one card at a time from any deck they choose, and they are free to switch from any deck to another at any time, and as often as they wish. However, the subject's decision to select from one deck versus another is largely influenced by various schedules 


\section{Behavioral Performance on $A^{\prime} B^{\prime} C^{\prime} D^{\prime}$}

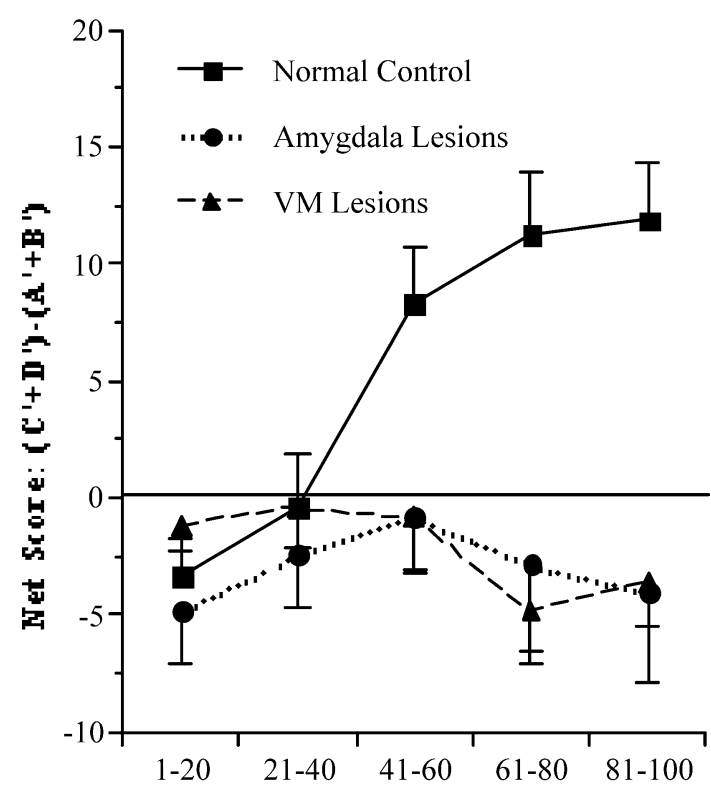

Blocks of Cards

Fig. 3. Relative to normal control subjects, patients with bilateral ventromedial prefrontal cortex lesions (VM lesions) or bilateral amygdala lesions (Amygdala Lesions) were impaired in their performance on the gambling task (GT). The figure shows net scores $\left(\left(C^{\prime}+D^{\prime}\right)-\left(A^{\prime}+B^{\prime}\right)\right)$ of cards selected by each group across different blocks expressed as mean + s.e.m. Positive net scores reflect advantageous performance while negative net scores reflect disadvantageous performance.

of immediate reward and future punishment. These schedules are pre-programmed and known to the examiner, but not to the subject. The reward/punishment schedules are set in such a way so that two of the decks of cards $(A$ and $B$ ) yield high immediate gain but larger future loss, i.e. long term loss (disadvantageous decks), and two of the decks $(C$ and $D)$ yield lower immediate gain but a smaller future loss, i.e. a long term gain (advantageous decks).

We investigated the performance of normal control subjects with demographic characteristics matched to a group of patients with bilateral damage to the ventromedial (VM) prefrontal cortex and a separate group of patients with bilateral damage to the amygdala. Normal subjects avoided the bad/disadvantageous decks ( $A$ and $B$ ) and preferred the good decks $(C$ and $D)$. By contrast, VM patients as well as amygdala patients did not avoid (i.e., they preferred) the bad decks ( $A$ and $B$ ) (Fig. 3). From these results we suggested that the VM and amygdala patients' performance profile is comparable to their real-life inability to decide advantageously (Bechara et al., 1999). But why do these patients behave in this disadvantageous manner? 


\subsection{Decision-making is a process guided by emotions}

The support for this notion comes from studies of patients with impaired decision making resulting from specific brain lesions, who showed abnormal activation of covert somatic/emotional signals that bias decisions in the advantageous direction. Indeed, the more pertinent evidence in support of the Somatic Marker Hypothesis and the reactivation of somatic signals related to prior experience is the failure to generate somatic signals when pondering decisions. This evidence comes from a study where we added a physiological measure to the gambling task. The goal was to assess somatic state activation while subjects were making decisions during the gambling task. We studied normal subjects, VM, and amygdala patients. We had them perform the gambling task while we recorded their SCRs (Bechara et al., 1999).

Normal subjects, generated SCRs when they received reward or punishment. Most important, as they became experienced with the task, they began to generate SCRs prior to the selection of any cards, i.e., during the time when they were pondering from which deck to choose (Fig. 4). These anticipatory SCRs were more pronounced before picking a card from the risky decks $A$ and $B$, when compared to the safe decks $C$ and $D$. The VM patients generated SCRs to reward or punishment, albeit the responses were slightly lower than those from normal controls, but the amygdala patients completely failed to generate SCRs in reaction to reward or punishment. Furthermore, the VM as well as the amygdala patients entirely failed to generate SCRs before picking a card (Fig. 5).

These results suggest that when the amygdala is damaged, the patient can no longer register how painful it feels when one loses money. This in turn "misleads" the VM cortex regarding how painful it should feel if a decision led to money loss. Together, the results provide support for the notion that decision-making is guided by emotional (somatic) signaling generated in anticipation of future events. Without the ability to generate these emotional signals, the patients fail to avoid the decks that lead to painful losses, and instead they sample the wrong decks until they go broke in a manner that is very similar to how they behave in real life. Thus both emotional parts of the brain, the amygdala and VM cortex assist with rational decisions.

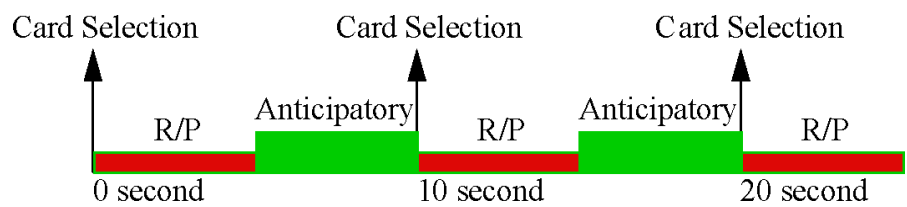

Fig. 4. The time interval between two consecutive card selections was subdivided into two time windows for measuring skin conductance responses (SCRs), a physiological measure under autonomic nervous system control, which we used as an index of the activation of somatic states. The time window ( 5 seconds in length) immediately following the selection of a card was called Reward/Punishment (R/P), because the SCRs generated during this time window were in reaction to the outcome of winning or losing a certain amount of money. The time window preceding the selection of the next card was called Anticipatory, i.e., the time during which the subject was pondering which card to select. 


\section{REWARD and PUNISHMENT SCRs}

Advantageous Decks (C\&D)

Disadvantageous Decks (A\&B)

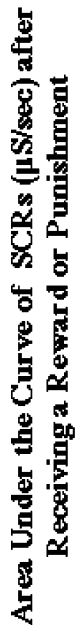

0.2

-

0.2

0.2

0.1

0.1

0.1

0.1

0.1

0.0

0.0

0.0

0.0

0.0

Reward

Normal

Amygdala

VM

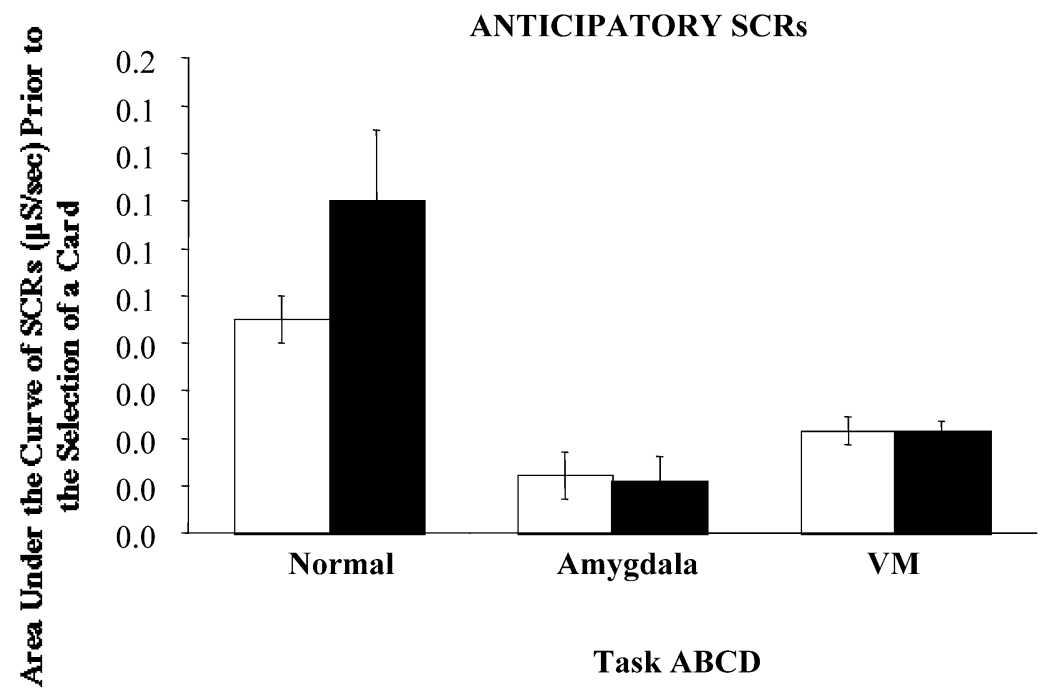

Fig. 5. Means + s.e.m. of reward and punishment SCRs (top panel), and anticipatory SCRs (bottom panel), shown in $(\mathrm{S} / \mathrm{sec})$, which were generated by controls, amygdala, or VM patients in association with the advantageous decks $(C$ and $D)$ versus the disadvantageous decks $(A$ and $B)$. 


\subsection{Conscious knowledge alone is not sufficient for making advantageous decisions}

In follow-up investigations, we showed that somatic signals generated in anticipation of future outcomes do not need to be perceived consciously. We carried out an experiment similar to the one above, where we tested normal subjects and VM patients on the gambling task, while recording their SCRs. However, once the subject had picked 10 cards from the decks, we stopped the game briefly, and asked the subject to declare whatever they knew about what was going on in the game (Bechara et al., 1997). From the answers to the questions, we were able to distinguish four periods of performance as subjects went from the first to the last trial in the task.

The first was a "pre-punishment" period, when subjects sampled the decks, before they had yet encountered any punishment. The second was a "pre-hunch" period, when subjects began to encounter punishment, but still had no clue about what was going on in the game. The third was a "hunch" period, when subjects began to express a hunch about which decks were riskier, but they were not certain. The fourth was a "conceptual" period, when subjects knew very well that there were good and bad decks, and which decks were good and bad.

When we examined the anticipatory SCRs from each period, we found that, in the normal subjects, there was no significant activity during the pre-punishment period. There was a substantial rise in anticipatory responses during the pre-hunch period, i.e., before any conscious knowledge developed. This SCR activity was sustained for the remaining periods. When we examined the behavior during each period, we found that there was a preference for the high paying decks $(A$ and $B)$ during the pre-punishment period. Furthermore, there was a hint of a shift in the pattern of card selection, away from the bad decks, even in the pre-hunch period. This shift in preference for the good decks became more pronounced during the hunch and conceptual periods. The VM patients on the other hand, never reported a hunch about which of the decks were good or bad. Furthermore, they never developed anticipatory SCRs, and they continued to choose more cards from decks $A$ and $B$ relative to $C$ and $D$ (Fig. 6).

Also, even though $30 \%$ of controls did not reach the conceptual period, they still performed advantageously. Although 50\% of VM patients did reach the conceptual period, they still performed disadvantageously (Bechara et al., 1997) (Fig. 7). These results show that VM patients continue to choose disadvantageously in the gambling task, even after realizing the consequences of their action. This suggests that these anticipatory SCRs are an index of activated unconscious biases derived from prior experiences with reward and punishment. These biases help deter the normal subject from pursuing a course of action that is disadvantageous in the future. This occurs even before the subject becomes aware of the goodness or badness of the choice s/he is about to make. Without these biases, the knowledge of what is right and what is wrong may still become available. However, by itself, this knowledge may not be sufficient to ensure advantageous behavior. Therefore, although the VM patient may become fully aware of what is right and what is wrong, he fails to act accordingly. Thus they may "say" the right thing, but they "do" the wrong thing.

Thus "knowledge" without "emotional signaling" leads to dissociation between what one knows or says, and how one decides to act. This dissociation is not restricted to neuro- 
Normal

Ventromedial Prefrontal

\section{PERIOD}

\begin{tabular}{|c|c|c|c|c|c|c|}
\hline $\begin{array}{l}\text { Pre- } \\
\text { punishment } \\
\text { (Baseline) }\end{array}$ & $\begin{array}{l}\text { Pre- } \\
\text { Hunch }\end{array}$ & Hunch & $\begin{array}{l}\text { Conceptual } \\
\text { Period }\end{array}$ & $\begin{array}{l}\text { Pre- } \\
\text { punishment } \\
\text { (Baseline) }\end{array}$ & $\begin{array}{l}\text { Pre- } \\
\text { Hunch }\end{array}$ & $\begin{array}{l}\text { Conceptual } \\
\text { Period }\end{array}$ \\
\hline
\end{tabular}

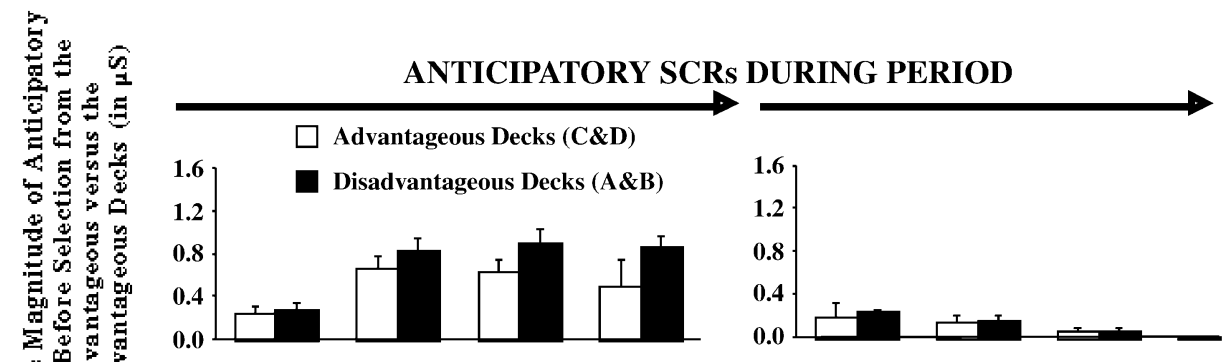

0.0

\section{BEHAVIORAL RESPONSES DURING PERIOD}
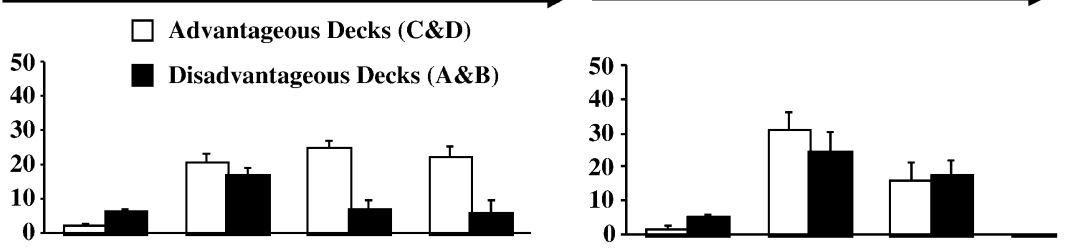

\% SUBJECTS

REACHING

PERIOD

$$
100 \% \quad 100 \% \quad 100 \% \quad 70 \%
$$

$100 \% \quad 100 \%$

$\mathbf{5 0 \%}$

Fig. 6. Presentation of the four periods in terms of average numbers of cards selected from the bad decks $(A$ and $B)$ versus the good decks $(C$ and $D)$, and the mean magnitudes of anticipatory SCRs associated with the same cards.

logical patients, but it also applies to neuropsychiatric conditions with suspected pathology in the VM cortex or other components of the somatic marker circuitry:

(1) addiction, in which patients know the consequences of their drug seeking behavior, but still take the drug, and

(2) psychopathy, in which the individuals are also aware of the consequences of their actions, but still plan and execute the killing or rape of a victim.

\subsection{The implementation of decisions under certainty engages different neural circuitry than that of decisions under uncertainty or ambiguity}

Somatic markers may influence decisions via a "body loop" or "as-if-loop" as explained earlier (Fig. 2). When do decisions engage the "body loop" or the "as-if-loop"? 

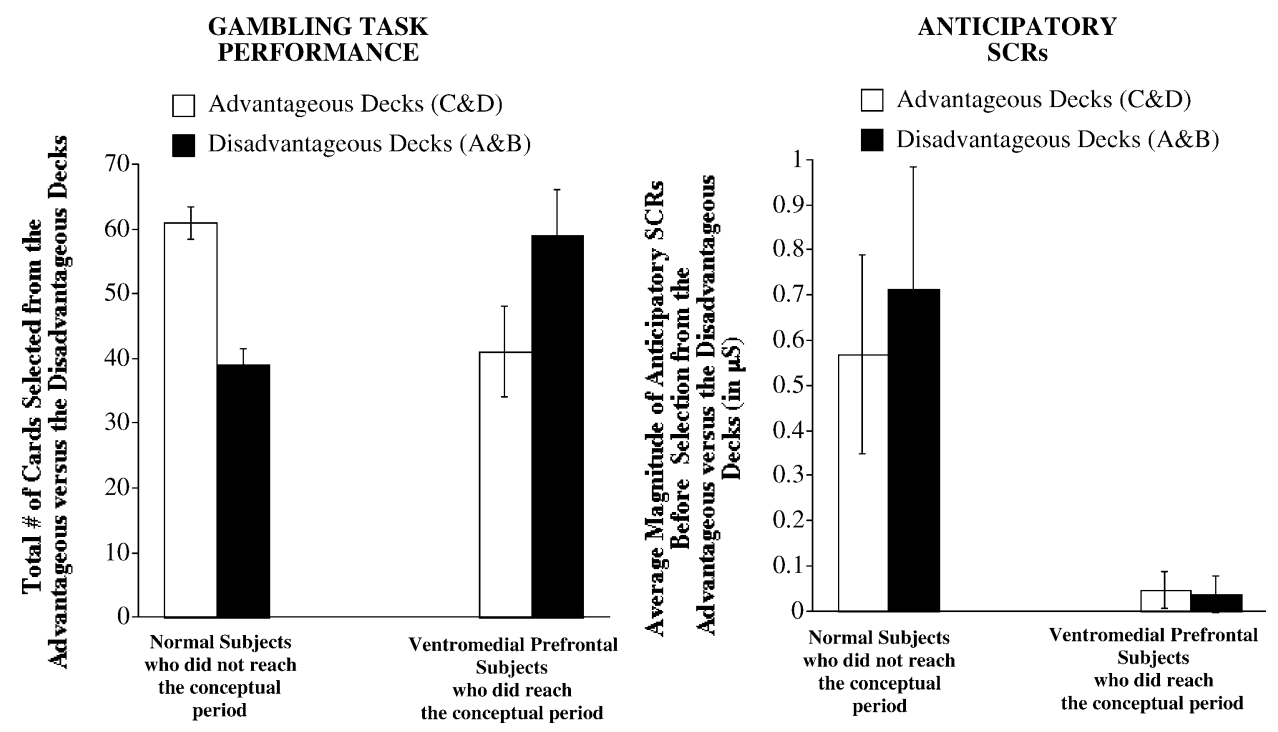

Fig. 7. Presentation of the choices made by normal and VM patients who did or did not reach the conceptual period in terms of average numbers of cards selected from the bad decks ( $A$ and $B$ ) versus the good decks $(C$ and $D)$, and the mean magnitudes of anticipatory SCRs associated with the same cards.

The answer to this question is being investigated, but a preliminary study is available. Behavioral economists describe 3 classes of choice:

(1) choice under certainty,

(2) choice under risk, and

(3) choice under ambiguity (Einhorn and Hogarth, 1985; Ellsberg, 1961).

Preliminary studies using the gambling task (a task that measures decisions ranging from high uncertainty to ambiguity) and the "betting" task (a task that measures decisions ranging from low uncertainty (e.g., 90\% certain) to high uncertainty (e.g., 50\% certain)), support the hypothesis that the "body loop" mode of operation becomes increasingly prominent as decisions move from certainty to risk, and to ambiguity. Rogers and his colleagues (Rogers et al., 1999a) developed a decision-making task, the "betting" task, which was shown to be sensitive to orbitofrontal lobe damage. Functional neuroimaging studies using the same task revealed increased activation in the orbitofrontal region, right parietal cortices, and uncus (overlapping the amygdala) (Rogers et al., 1999b), all areas that include the target regions that we hypothesize as critical for decision-making. However, there is a fundamental difference between the "betting" task and our gambling task. In the gambling task, subjects are not explicitly told the pay-off structure. Rogers suggested that the lack of specified contingencies would make it difficult to characterize the underlying deficit. The idea was that at least in some cases, a person makes disadvantageous choices because s/he is failing to take long-range interests into account, or because $\mathrm{s} / \mathrm{he}$ is unaware of the actual contingencies. This was one of the reasons why Rogers and 
colleagues designed the "betting" task to measure deficits in orbitofrontal functioning. In the "betting" task, subjects are asked to decide among choices in which odds explicitly favor one of the available options. While the outcomes of individual trials may not be $100 \%$ certain, and according to the labels mentioned earlier this would be called a task of risk, the level of certainty ranges from very high (i.e., 90\%) to somewhat risky (i.e., 60\%). By contrast, in the gambling task, which according to the labels mentioned earlier would be called a task of ambiguity, the level of uncertainty remains high throughout. Subjects never acquire knowledge about the probabilities of reward and punishment, even when they reach conceptual knowledge about the overall goodness and badness of the various choices. Preliminary findings indicate that normal subjects generate minimal anticipatory SCRs during the "betting" task, especially in relation to the most certain choices compared to the most risky. Most important, the overall average of anticipatory SCRs generated during the "betting" task are lower than those from the gambling task, consistent with the idea that decision-making under ambiguity, where the outcome is unknown, unpredictable, and cannot be estimated, engages the "body loop." By contrast, decision-making under certainty, where the outcome is explicit and predictable, engages the "as-if body loop."

\subsection{Emotion may not always be beneficial to decision-making}

Although the somatic marker view argues that emotions are an important factor in the process of decision-making, there is a popular notion that "emotions cloud the mind and interfere with good judgment," and that "wise decisions and judgments come only from cool heads." How can we reconcile these seemingly conflicting views? Do emotions help the process of making advantageous decisions or disrupt it?

The somatic marker hypothesis concerns emotion that is integral to the decision-making task at hand. For instance, when deciding to speed on a highway because you are late for an interview, the "thought" of being stopped by a police, or the "thought" of getting into an accident will trigger somatic states (e.g., some form of a fear response). However, these somatic states are integral to the decision-making task at hand, i.e., the decision on whether to speed or not. These somatic states are indeed beneficial, because they consciously or non-consciously bias the decision in an advantageous manner. However, the induction of somatic states that are unrelated to the decision task at hand (for example receiving a cell phone call about someone dying in the family while driving) may become disruptive.

Support of this hypothesis comes indirectly from clinical observations of neuropsychiatric patients with bipolar disorders, who show disturbances in decision-making that include indecisiveness (during depression) or impulsiveness (during mania) (First et al., 1997). Experimental evidence also suggests that the presence of such unrelated emotions shifts decisions in the direction of short-term goals (Gray, 1999). Preliminary evidence in normal subjects suggests that the induction of strong emotional states (e.g., by the recall of personal emotional experiences) prior to the performance of the gambling task, reduced the number of choices from the advantageous decks (Fig. 8). 


\section{Average Gambling Task Performance Following Nuetral Imagery vs. Emotion Imagery}

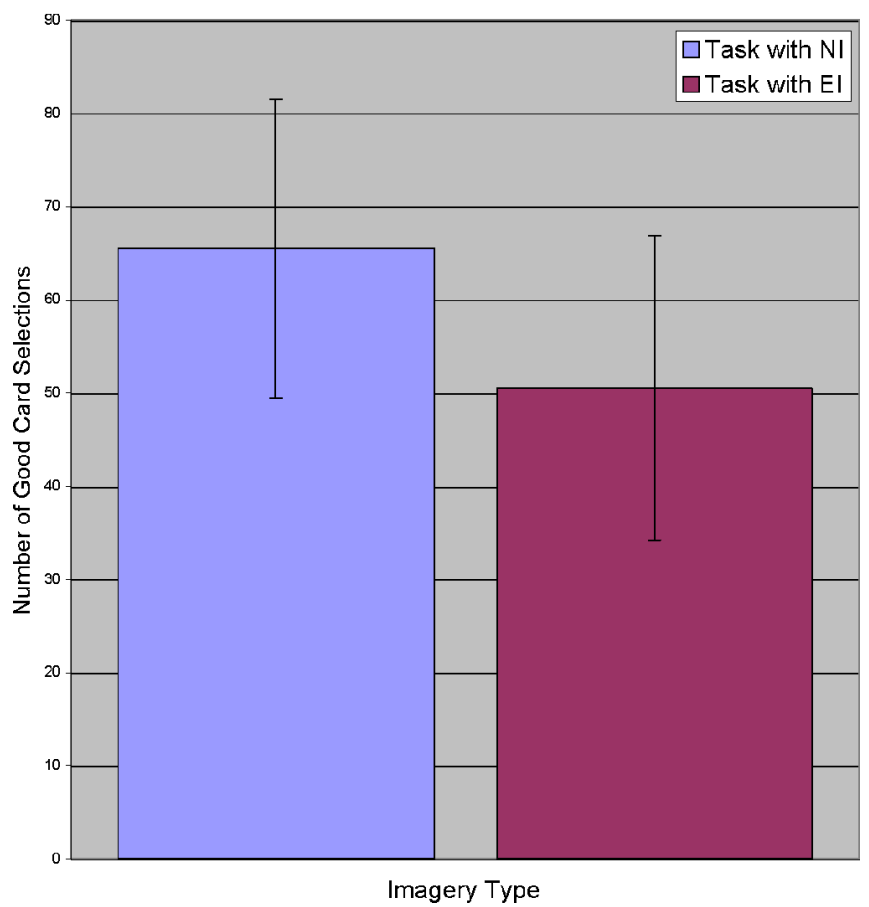

Fig. 8. Average number of card selections from the advantageous decks from ten healthy volunteers, each of whom performed the gambling task after recalling a neutral emotional event (Task with NI (neutral imagery)), such as mowing the lawn, and after recalling an emotional event (Task with EI (emotional imagery)), such as recalling the death of a loved one. The order of neutral versus emotional induction was counterbalanced among subjects.

\section{Application of the somatic marker hypothesis to economic decisions}

Most theories of economic choice are cognitive in perspective and assume that decisions derive from an assessment of the future outcomes of various options and alternatives through some type of cost-benefit analysis (see Loewenstein et al., 2001 for a review). There are exceptions, i.e., a few theories that addressed emotion as a factor in decisionmaking (Janis and Mann, 1977; Mann, 1992), however, they address emotions that are the consequence of some decision (e.g., the disappointment or regret experienced after some risky decision that worked out badly), rather than the affective reactions arising directly from the decision itself at the time of deliberation. The somatic marker hypothesis provides neurobiological evidence in support of the notion that people often make judgments based on "hunches," "gut feelings," and subjective evaluation of the consequences (Damasio, 1994; Loewenstein et al., 2001; Schwartz and Clore, 1983; Tversky and Kahneman, 1981; Zajonc, 1984). 


\subsection{The amygdala}

Exposure to primary inducers triggers somatic states via the amygdala system that are fast, automatic, and obligatory. Somatic states triggered by the amygdala are short lived and habituate very quickly (Buchel et al., 1998; Dolan et al., 1996; LaBar et al., 1998). In other words, primary inducers trigger an emotion via the amygdala quickly, without much thought and effort, and before one can figure out what just happened.

Evolutionarily, the amygdala has probably evolved to embrace one instantly for a "fight or flight" response, and it has evolved during a time when probably there was no harm in confusing false alarms and real ones: if the amygdala sent a person running away from what looked like a snake, the person was safe! Even if this turned out to be a false alarm, e.g., a wood stick that looked like a snake, the person was still safe, and there was no harm in responding to a false alarm (LeDoux, 1996). However, in the world of economics, panicky responses to false alarms - the rush to sell as one sees the stocks dropping - can be very costly; perhaps this is a situation where it is not so great to respond to false alarms (Zweig, 2002). However, this does not mean that the amygdala is disruptive to economic decisions. The amygdala has evolved for a survival purpose, and it did not have the stock market in mind. The automatic emotions triggered by the amygdala are generally beneficial and serve an adaptive role in life.

Specifically, the normal acquisition of secondary inducers requires the integrity of the amygdala, and also the somatosensory neural system conveying somatic signals from the internal milieu and viscera, via the brainstem, to the insular/SII, SI cortices. When the amygdala, or critical components of the somatosensory system, is damaged, then primary inducers cannot induce somatic states, or signals from triggered somatic states cannot be transmitted to somatosensory cortices. Consequently, secondary inducers cannot acquire somatic state representations. The consequence is illustrated in the gambling task experiment described earlier. When the amygdala is damaged, the patient can no longer signal how painful it feels when one loses money. This in turn fools the VM cortex about how painful it should feel if a decision led to money loss. Without the ability to generate these emotional (somatic) signals, the patients fail to avoid the decks that lead to painful losses, i.e., they sample the wrong decks until they go broke, in a manner that is very similar to how they behave in real life.

Thus the panicky response of the amygdala when one sees the stocks dropping is an adaptive response that serves a beneficial role in market decisions, even when it appears that it is not doing so.

\subsection{The VM cortex}

Secondary inducers trigger somatic states via the VM cortex from perceived or recalled mental images. These somatic states may become conscious (i.e., perceived as a good or bad feeling) or remain non-conscious. While the amygdala is engaged in emotional situations requiring a rapid response, i.e., "low-order" emotional reactions arising from relatively automatic processes (Berkowitz, 1993; LeDoux, 1996), the VM cortex is engaged in emotional situations driven by thoughts and reflection. Once this initial amygdala emotional response is over, "high-order" emotional reactions begin to arise from relatively 
more controlled, higher order processes involved in thinking, reasoning, and consciousness (Schneider and Shiffrin, 1977). Unlike the amygdala response, which is sudden and habituates quickly, the VM response is deliberate, slow, and lasts for a long time.

Thus the prefrontal cortex, especially the VM part, helps predict the emotion of the future, thereby forecasting the consequences of one's own actions. However, the VM cortex is relatively a large region of the brain, and it has developed throughout evolution, so that not every part performs the same function. We suggest that the mechanisms by which different "thoughts" or "mental representations" are coupled to somatic states via the VM region are based on hierarchical functional organization of the VM cortex in relation to time and probability. This organization is rooted in evolution, and perhaps it explains several aspects of human economic choice.

\subsubsection{Somatic states and time}

Several human and animal studies have suggested that the prefrontal cortex is involved in the memory of time, and that there are neurotransmitter systems (e.g., dopamine and acetylcholine) differentially involved in timing (e.g., see Fuster, 1996; Nichelli, 2002 for a review). However, the processing of time can be complex, and some authors have proposed that time can take several different forms, i.e.,

(1) temporal order, which refers to sequential occurrence of events,

(2) time duration, which refers to the memory of intervals between events, and

(3) time perspective, which involves the memory for anticipating future events (Nichelli, 2002).

A number of lesion studies in animals and humans have linked these memory functions to the dorsolateral sector of the prefrontal cortex (Milner et al., 1985, 1991; Petrides, 1985, 1993; Shimamura et al., 1990).

We propose that evoking each of these memories for time in the dorsolateral prefrontal cortex (i.e., secondary inducers) will activate a somatic state representation. Thus the neural cells of the VM region are specialized to simply couple

(1) the neural ensemble holding a memory representation of time with

(2) the appropriate somatic state.

However, the coupling of different categories of memories (or secondary inducers) to their somatic states is hierarchically organized: from interactions of cells that respond to various components of time, especially time duration emerge representations of events that are more immediate or more distant in the future. Human lesion studies suggest that representations of outcomes or consequences that are near in time recruit more caudal/posterior VM cortices, whereas representations of outcomes/consequences that are far in time recruit more rostral/anterior VM cortices. For instance, patients with bilateral VM lesions, especially those with lesions that spare the posterior VM region and involve only the more rostral/anterior areas, demonstrate deficits in somatic state activation that are selective for domains involving the remote future, i.e., they have "myopia" for consequences that will occur in the far, as opposed to the more immediate, future (Damasio, 1994). 
This hierarchical organization is rooted in evolution. The memory for time is evident in rats and pigeons (see Nichelli, 2002 for a review). However, the functional evolution of the prefrontal cortex appears to involve an incremental increase in its capacity to access representations of events that occur in the more distant future. This enhanced "futuristic" capacity coincides with the development of more rostral/anterior regions of the VM cortex. Comparative studies of the frontal lobes in humans and non-human primates have revealed that the major advancement in the size, complexity, and connectivity of the frontal lobes in humans relates primarily to BA 10, i.e., the frontal pole (Semendeferi et al., 2001), and not so much to the more posterior areas of the VM cortex (Semendeferi et al., 2002).

Time may also be represented in the form of "how many steps" one needs to take, and in "what order" these steps must be, before attaining the ultimate goal. We suggest that from different interactions of cells that respond to various aspects of time, especially temporal order and time perspective, emerge representations of future outcomes/consequences that are more concrete or tangible, i.e., the outcome is directly associated with an actual reward/punishment; for instance, money is a more tangible item because it can directly secure food, water, shelter, and so on. There are also representations of outcomes that are more $a b$ stract, i.e., there are so many levels of learning associations between the next outcome and the actual reward/punishment; for instance, there are several layers of associations between working hard to receive a good grade on an exam and an actual reward, such as receiving a diploma first, getting a good job, earning money, and then securing food, water, shelter, and so on. Human studies suggest that representations of outcomes or consequences that are more concrete/tangible recruit more caudal/posterior VM cortices, whereas representations of outcomes/consequences that are more abstract recruit more rostral/anterior VM cortices. Patients with bilateral VM lesions, especially those that spare the posterior VM region, exhibit decision-making impairments in domains with consequences that are less concrete/tangible. For instance, they make choices that lead several steps down the line to financial losses, the loss of friend and family relationships, but they never engage in actions that immediately lead to physical harm to themselves or to others (Damasio, 1994). Lesions of the same VM area during childhood impairs the development of moral and ethical judgment, i.e., making judgment in more complex and abstract situations, as opposed to making judgment in more concrete situations such as causing bodily harm to themselves or others (Anderson et al., 1999). Functional neuroimaging studies involving the solution of moral dilemmas and making ethical decisions have shown increased activity in the more rostral sectors of the VM cortex, i.e., the frontal pole (Greene et al., 2001).

Again, this hierarchical organization is rooted in evolution. The memory for temporal sequencing is evident in rats. Paradigms of "second order conditioning" and "occasion setting" have addressed the issue of learning "how many responses" and "in what order" an animal needs to perform before obtaining a real reward. These studies have implicated the prefrontal cortex of the rat in these forms of learning (Gallagher et al., 1999; Schoenbaum et al., 1998). However, the degree of "abstractness" can be increased indefinitely, and that's when the differentiation between animals and humans begin to emerge. Although second order, and perhaps third order, conditioning can be achieved in laboratory rats, higher orders of conditioning and learning associations have not been reported. Non-human primates can acquire several degrees of higher level learning associations than 
rats, but this learning does not even approach the level of complexity that humans can achieve. We suggest that the increased capacity of humans to cope with abstraction (i.e., activate somatic states from concepts that are so many steps removed from obtaining a real reward/punishment) coincides with the development of more rostral/anterior regions of the VM cortex as indicated earlier.

\subsubsection{Somatic states and probabilities/frequencies}

Several human and animal studies have suggested that the prefrontal cortex is involved in the recognition or estimation of frequency (Milner et al., 1991; Smith and Milner, 1984). Other work has implicated the anterior cingulate in the recognition of patterns (e.g., repetition and alternation) (Huettel et al., 2002). Although it can be argued that probabilities and frequencies can be reduced to another component of time, the two are conceptually different. We suggest that representations of future outcomes/consequences that are more or less probable (or more or less expected) emerge from interactions of cells that respond to probabilities/frequencies. Human studies suggest that representations of outcomes/consequences that are more predictable recruit more posterior VM cortices (including anterior cingulate), whereas representations that are less predictable recruit more anterior cortices. For instance, patients with bilateral VM lesions undergoing Pavlovian conditioning (i.e., the conditioned stimulus is expected $100 \%$ of the time to be followed by the aversive unconditioned stimulus) generated somatic responses in anticipation of the unconditioned stimulus (Bechara et al., 1999). By contrast, during the gambling task (i.e., when the punishment occurs $10 \%$ or $50 \%$ of the time), the same patients failed to generate anticipatory somatic responses, i.e., they failed to generate somatic responses when punishment was less predictable (Bechara et al., 1996). Most intriguing, patients with VM lesions that were more posterior (i.e., extended to the anterior cingulate and basal forebrain), they also failed to acquire anticipatory somatic responses during Pavlovian conditioning, as well as the gambling task (Tranel et al., 1996), thus consistent with the hypothesis that representations of future outcomes that are highly probable recruit posterior VM cortices, whereas representations of outcomes/consequences that are less probable recruit anterior VM cortices.

This ability to detect and estimate probabilities/frequencies is rooted in evolution. There are differences in the behavioral responses, as well as the brain mechanisms, of animals and humans when dealing with probabilities and frequencies (Wolford et al., 2000). We suggest that the functional evolution of the VM cortex involved an incremental increase in its capacity to respond to events that are less frequent or probable. This enhanced sensitivity of human VM cells to respond to events with low probability of occurrence coincides with the development of more rostral/anterior regions of the VM cortex.

\subsubsection{Somatic states and valence}

"It is an old assumption in psychology that every experience falls somewhere along a hedonic continuum, and that positive or negative feelings are evoked by most (if not all) words and objects in an automatic fashion, very quickly, without conscious control and not infrequently without awareness" (Overskeid, 2000). The question becomes which neural cells in the VM region trigger or access positive versus negative somatic states?

Numerous studies have argued that the right hemisphere plays a dominant role in experiencing unpleasant feelings, whereas the left hemisphere is important for pleasant feelings 
(Davidson and Irwin, 1999; Deglin and Kinsbourne, 1996; Overskeid, 2000). Although this issue is not settled, we suggest that representations of outcomes/consequences that are positive are coupled to positive somatic states via predominantly left VM cortices. Representations of events that are negative are processed via predominantly right VM cortices.

A partial support for this hypothesis comes from evidence that unilateral damage to the right VM cortex resulted in "myopia" for future negative consequences. Patients with left VM damage were less (or not) impaired in this domain (Manes et al., 2002; Tranel et al., 2002). It remains to be seen whether the impairment in the left VM patients relates to "myopia" for future positive consequences.

\subsubsection{Somatic states and magnitude}

Anatomically, the more caudal/posterior areas of the VM cortex (e.g., BA 25) are directly connected to brainstem structures for triggering and/or representing somatic states (e.g., autonomic, neurotransmitter, and sensory nuclei), and to cortical structures holding conscious representations of somatic states (i.e., "what it feels like") in insular/SII, SI cortices (Ongur and Price, 2000). By contrast, the connections of more rostral/anterior areas of the VM cortex to neural structures involved in triggering and/or accessing representations of somatic states are more indirect. It follows that coupling of information (secondary inducers) to representations of somatic states via posterior VM cortices is relatively fast, effortless, and strong. In contrast, coupling of secondary inducers to somatic states via anterior VM cortices is relatively slow, effortful, and weak.

It is important to clarify here an important point. Events (secondary inducers) that are highly probable (i.e., almost certain) are processed by more posterior VM cortices, they trigger stronger somatic states relative to less probable ones. However, we have suggested earlier that decision-making under certainty engages the "as-if-body loop," whereas decision-making under ambiguity engages the "body loop." This should not be taken to mean that "as-if-body loop" activation results in stronger somatic states than "body loop" activation. Furthermore, somatic states detected in the body are not necessarily an indicator that the somatic state is stronger than if it were triggered intra-cerebrally. We believe that the difference between the two modes of operation of somatic states reflects the complexity of the mechanisms for triggering somatic states as opposed to the strength or magnitude of somatic states. In decisions under certainty, response options (secondary inducers) are limited, and accessing somatic state representations is straightforward, i.e., fast, effortless, and it can be very strong. For example, walking into a bank and finding a million dollars on a table does not require triggering somatic states in the body (body loop) in order to suppress any impulse to take the money. The impulse is suppressed quickly and robustly. In contrast, in decisions under ambiguity, response options are numerous, complex, and conflicting. For example when finding a million dollars in a dark alley, deciding what to do with the money may indeed engage the body loop. However, this is not a good indicator of whether the somatic state triggered in the body in this instance is stronger or weaker than that triggered inside the bank; the quality is different. Thus decisions under certainty or ambiguity, and engaging the "as-if-body loop" or "body loop" reflect different qualities or mechanisms of decisions as opposed to different strengths or magnitudes of somatic states (e.g., see Bechara, 2003 for a discussion of these different mechanisms). 


\subsubsection{Somatic states, the VM cortex, and economic decisions}

The anatomical and functional arrangement of the VM cortex provides a neurobiological explanation for several findings of economic studies on consumers' choice.

First, is the issue of delayed discounting, so that $\$ 1000$ tomorrow is preferred to $\$ 2000$ two years from now (Green et al., 1994a, 1994b; Herrnstein and Prelec, 1991; Kirby and Herrnstein, 1995). Similarly, losing a \$1000 tomorrow hurts more than losing \$2000 two years from now. The organization of the VM region in relation to time (i.e., "near future" is processed more posteriorly, whereas "distant future" is more anterior) explains why information conveying immediacy trigger stronger somatic responses, and therefore exert a stronger bias on decisions, than information conveying delayed outcomes.

The organization of the VM cortex along the axis of "concrete/tangible" to "abstract" may also explain why, for instance, people have an easier time spending money on credit cards as opposed to spending real money. Similarly, spending money becomes no object when a disease threatens the life of a loved one, and so on. This is because credit is more abstract than money, and money is more abstract than losing a "bond" from a loved one. Indeed, bonding is biologically innate; chicks bond to a mother figure almost after the first sight. It follows that losing a loved one (a more concrete/tangible secondary inducer that is processed more posteriorly) trigger stronger somatic responses then losing real money, and spending real money is more painful than spending credits, and so on.

Finally, is the issue of choice framing, based on the "Prospect theory" of Kahneman and Tversky (Tversky and Kahneman, 1974, 1981). The theory suggests that individuals are irrational decision makers, which is contrary to popular belief. The authors showed that decision makers exhibit a willingness to take bigger gambles (risk seeking) when faced with a loss situation, but they are quick to seize gains or the sure thing when also faced with a gamble (risk aversion). In other words, people simply do not like losses. For instance, people prefer a sure gain of $\$ 100$ to $50 \%$ probability of winning $\$ 200$ or nothing (i.e., they are risk averse in the face of sure gain). On the other hand, people avoid a sure loss of $\$ 100$ and take a chance on $50 \%$ probability of losing $\$ 200$ or nothing (i.e., they are risk seekers in the face of sure loss). This behavior contradicts current economic fundamentals that portray consumers as rational decision makers. The observations of Kahneman and Tversky were ingenious because they captured aspects of human economic choice that were contrary to the conventional wisdom. However, the theory did not explain why humans choose the way they do; the somatic marker model offers a neurobiological explanation for why information conveying sure outcomes trigger stronger somatic responses than information conveying less probable outcomes. Thus a sure gain of $\$ 100$ triggers a stronger somatic response than a probable gain of $\$ 200$, and a sure loss of $\$ 100$ triggers a stronger somatic response than a probable loss of \$200. A more important point here, which probably was not addressed in the Prospect Theory, is that "risk seeking" and "risk aversion" can be modulated by "background" somatic states, i.e., pre-existing somatic states triggered by prior economic events. The mechanisms that enhance or reduce "risk seeking" and "risk aversion" are discussed later. 


\subsection{Other mechanisms of somatic state activation and their role in economic decisions}

Wall Street strategists always attempt to predict where the market is headed, a behavior labeled "the prediction addiction" (Zweig, 2002). Why do these strategists attempt to predict the unpredictable? Why are their "hunches" and "gut feelings" often better predictors than the data that are sitting in front of them?

In a very intriguing article, Overskeid presented a convincing case of how having a problem creates a negative feeling state that the person wants to escape. When solving a problem, people always choose the solution that elicits the best feeling (Overskeid, 2000). Sometimes people choose a painful solution (e.g., accept that they have cancer and that they will die). However, choosing a painful solution is itself a problem, a state that one would rather escape, and when people accept a painful solution, they do so because all other available solutions are even more painful (Overskeid, 2000). Using Overskeid's conceptualization, not knowing where the market is headed is a problem, a negative state that one wants to escape. Predictions are solutions that elicit good feelings in the predictor, even though they may not be correct. Overskeid makes the argument that people offer the solutions that make them feel better, and not necessarily the solutions that they think they may be correct. Overskeid supports this argument with a quote from Dostoevsky, the deeply religious Russian author who once said: "Even if somebody proves to me that Christ does not exist, I would rather be with Christ than with the truth" (Overskeid, 2000). Thus "the prediction addiction" probably results from a constant drive to escape problems (not knowing where the market is headed) by selecting the solutions (i.e., predictions) that feel the best. The proposal by Overskeid is quite in line with the somatic marker hypothesis, except that the latter focuses more on the neurobiological mechanisms underlying feelings and their elicitation by "thoughts" (secondary inducers). Together, this may provide a neurobiological explanation for the "prediction addiction" and the persistent tendency of individuals to predict the utterly unpredictable.

Furthermore, the somatic marker hypothesis can explain why "hunches" and "gut feelings" are often better predictors than market data and fact sheets. Evidence suggests that the striatum and the anterior cingulate are involved in recognizing patterns and calculating probabilities. These two areas respond almost immediately to patterns that either repeat or alternate (Huettel et al., 2002; Zweig, 2002). The anterior cingulate begins to anticipate another repetition after a stimulus occurs only twice in a row. It takes a bit longer to respond predictably to an alternating pattern, i.e., about six iterations as opposed to two (Huettel et al., 2002; Zweig, 2002). Thus if the market was simply a reliable repeating or alternating pattern, then perhaps the anterior cingulate and striatum would be sufficient to predict the next outcome. However, when the information is so complex and the patterns are not so clear, our cognition may keep struggling explicitly to figure which strategy might be best, but our somatic signals are what implicitly or explicitly bias us towards the advantageous strategy. In other words, in situations of uncertainty and ambiguity, logic and conscious deliberation may offer certain choices, but somatic states, in the form of "hunches" or "gut feelings," help select the most advantageous response option. In Overskeid's terms, they help select the solution that feels the best.

Once somatic states induced by primary and/or secondary inducers are triggered, an overall positive or negative somatic state emerges. We suggest that the mechanisms that 
determine the nature of this overall somatic state are consistent with the principles of natural selection, i.e., survival of the fittest. In other words, numerous and often conflicting somatic states may be triggered at the same time, but stronger ones gain selective advantage over weaker ones. With each "thought" brought to working memory, the strength of the somatic state triggered by that "thought" determines whether the same "thought" is likely to recur (i.e., will be brought back to memory so that it triggers another somatic state that reinforces the previous one), or whether that "thought" is likely to be eliminated. Thus over the course of pondering a decision, positive and negative somatic markers that are strong are reinforced, while weak ones are eliminated. This process of elimination can be very fast. Ultimately, an overall, more dominant, somatic state emerges (a "gut feeling" or a "hunch," so to speak), which then provides signals to the telencephalon that modulate activity in neural structures involved in biasing decisions.

In order for somatic signals to exert a "biasing" effect on behavior and on "thought," they must act on appropriate neural systems. Both the striatum and the anterior cingulate play a role in this biasing function.

\subsubsection{The striatum}

Evidence suggests that at the level of the striatum, the biasing mechanism of behavioral response selection is non-conscious, i.e., the subject learns to select a correct response, but without awareness of whether the response is right or wrong. There are several lines of evidence that support the notion that at the level of the nucleus accumbens/striatum the biasing action of somatic states is implicit or non-conscious:

(1) On a task called "the weather forecast task," Knowlton and Squire (Knowlton et al., 1996) showed that normal and amnesic subjects implicitly learned to predict the weather without awareness of the complex rules governing performance of the task. The behavioral guidance that occurred without awareness of the rules of the task was absent in subjects with Parkinson disease (PD), who did poorly on this task. However, as soon as PD subjects acquired an explicit knowledge of the rules governing the task, they began to improve their performance, i.e., behavioral guidance under the control of explicit knowledge was not impaired.

(2) Patients whose brain damage involves both medial temporal lobes, a portion of the orbital prefrontal cortex and the anterior cingulate, but spare the striatum/basal ganglia completely, demonstrated covert, but not overt, learning of affective valences (Tranel and Damasio, 1993).

(3) Berns, Montague, and their colleagues (Berns et al., 2001; Pagnoni et al., 2002) developed a task where subjects had to choose which of two buttons to press in order to get the maximum gain of $\$ 40$, by driving a slider all the way up to the top of a bar. In an fMRI scan, the investigators showed increased activity in the nucleus accumbens when subjects made the correct responses, while cognitively they were still struggling to figure out what pattern of button presses they should make. This experiment revealed a case of "knowledge without awareness."

These results suggest that the striatum is both necessary (Knowlton et al., 1996) and sufficient (Tranel and Damasio, 1993) to modify behavior through the influence of somatic states at a covert (implicit) level. We are currently examining the effects of striatum/nucleus accumbens lesions on the "pre-hunch/non-conscious" versus "post-hunch/conscious" pe- 
riod of the gambling task. Preliminary evidence reveals that subjects make the correct response, while cognitively they are still struggling to figure out which deck selections they should make. This instinctual recognition of the rewarding decks by the nucleus accumbens/striatum supports the hypothesized role of this region in "knowledge without awareness."

\subsubsection{The anterior cingulate}

At the level of the supracallosal sector of the anterior cingulate, and perhaps the adjacent supplementary motor area (SMA), the biasing mechanism of response selection is conscious, i.e., there is "action with awareness of what is right or wrong"; the decisions are "voluntary" or "willful," and guided by knowledge, awareness, and premeditation. Evidence shows that the anterior cingulate plays a role in the implementation of "voluntary" or "willful" decisions; decisions that are guided by "knowledge with awareness." Studies have shown that performance on target detection tasks and the Stroop interference task is associated with activity in the anterior cingulate (Pardo et al., 1990; Posner and Petersen, 1990; Posner et al., 1988). Another study (Frith et al., 1991) compared willed acts requiring explicit deliberate choice to automatic/routine acts and detected significant increase in activity in the supracallosal anterior cingulate during the willed acts. Another study (Petit et al., 1993) showed that the anterior cingulate region was activated during response selections associated with self-paced voluntary horizontal saccadic eye movements. These results suggest that the supracallosal anterior cingulate is involved in response selection when a wide range of novel choices is required, and when the response selection is carried at a conscious/explicit level.

\subsubsection{Other neural regions}

At the level of the lateral orbitofrontal and dorsolateral prefrontal region (LOF), the biasing mechanism of somatic states is conscious, but it is at the level of "thought" or "memory," and not the level of behavioral action. In other words, as one is pondering several options and scenarios in their working memory, the biasing effect of somatic states is to endorse some options and reject other ones, before any of these options are translated into actions.

\subsubsection{Pharmacological mechanisms of decision-making}

Evidence also suggests that the biasing action of somatic states is mediated through the release of neurotransmitters. Our work in humans suggests that, at least, both DA and 5-HT are implicated in the biasing effects of somatic states. DA biases decisions covertly (perhaps through action in the striatum). On the other hand, 5-HT biases decisions overtly (perhaps through action in the anterior cingulate and probably the adjacent SMA) (Bechara et al., 2001). The biasing effect of somatic states is also at the level of working memory in the LOF/DL prefrontal cortex, but the neurotransmitter system(s) that mediates this biasing function remains to be determined (Fig. 9). 


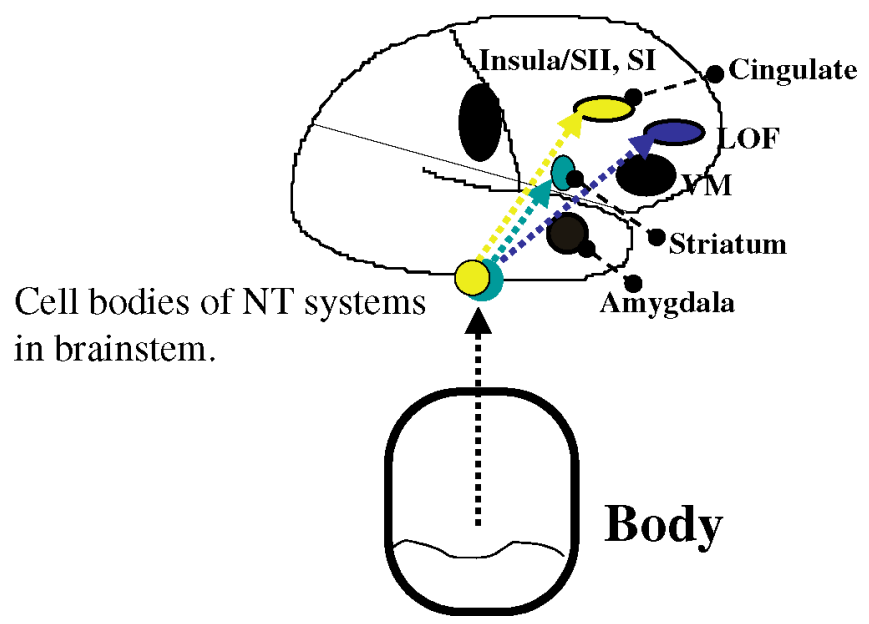

Fig. 9. A diagram illustrating three different levels at which somatic states can bias decisions via the release of neurotransmitters (NT). (1) Dopamine biases decisions covertly (perhaps through action in the striatum and affective sector of anterior cingulate (BA 25 and lower 24,32)). (2) Serotonin biases decisions overtly (perhaps through action in the cognitive sector of anterior cingulate and probably the adjacent SMA (Supplementary Motor Area)). (3) Somatic states also bias working memory in the LOF (lateral orbitofrontal and dorsolateral regions of the prefrontal cortex). They help endorse or reject "thoughts," "options," or "scenarios" brought to mind during the pondering of decisions, i.e., before their translation into action. The neurotransmitter system that mediates this biasing function remains to be determined.

\section{Neuroeconomics: a somatic marker model for predicting investors' choices}

The "Expected Utility Theory" and its followers described human choice in behavioral terms. For instance, in "The Theory of Games and Economic Behavior," von Neumann and Morgenstern (1944) described consumers as rational economic actors that select alternative options with the highest expected utility or value. The "Prospect theory" casts a doubt over this long-standing normative view of economic decision-making (Tversky and Kahneman, 1974, 1981). Other theorists have introduced psychological processes, such as personality characteristics, as important factors in explaining consumer's choice. For instance, Abramson, Seligman, and Teasdale (Abramson et al., 1978) proposed a psychological model of attribution processes, which provides a theoretical framework to view optimism and pessimism, and how individuals respond to negative circumstances and how they explain the causes of events. Other studies found that "Prospect theory" is more accurate when considering economic decisions of those individuals with an optimistic explanatory style; pessimists do not behave as well in accordance with the theory. However, none of these theories have addressed the neurobiological mechanisms underlying these market behaviors. For example, why do states of optimism lead to different choices than states of pessimism? Why when the market is crashing everyone rushes to sell, and when it is growing, every one rushes to buy? Why in investors who get a streak of several gains in the row, and then hit a loss, their panicky responses send them rushing to sell? The neurobiological mechanisms of somatic marker activation and decision-making help explain and predict some of these behavioral phenomena. 


\section{1. "Background" somatic states}

In the investment world, reactions to market news (primary inducers), as well as "thoughts" about what to do next (secondary inducers) induce somatic states. However, pre-existing somatic states influence the feeling and triggering of subsequent ones. Thus prior emotional events influence future economic choices.

\subsubsection{Positive and negative somatic states are physiologically distinguishable}

The somatic marker hypothesis posits that when pondering a decision, separate thoughts (secondary inducers) trigger a positive or negative somatic state. Depending on the relative strengths (magnitudes) of negative versus positive states, an overall somatic state will emerge that is either positive or negative. Evidence shows that positive and negative somatic states induce distinct physiological patterns that can be detected in laboratory settings as changes in heart rate, skin conductance, respiration, and so on (Cacioppo et al., 2000). This suggests that the brain discriminates between positive and negative somatic states signals, which different signals then exert different effects on decisions.

\subsubsection{Operation of the somatic marker circuitry is a complete circle}

The insular/SII, SI cortices are necessary although they may not be sufficient for feelings of emotion to occur (Damasio, 1999). On the other hand, the amygdala is a critical structure for triggering somatic states from primary inducers and the VM cortex is critical for secondary inducers. Feedback signals from pre-existing somatic states modulate activity in the neural structures that are critical for feeling and triggering somatic states, thereby influencing subsequent somatic states. Indeed, operation of the somatic marker circuitry is a complete circle: primary and secondary inducers trigger somatic states; feedback signals from triggered somatic states influence activity in neural structures critical for primary and secondary induction; the modulated neural activity within these structures will in turn influence subsequent induction of somatic states from primary and secondary inducers.

More specifically, pre-existing somatic states from streaks of losses or gains influence neurotransmitter release (e.g., dopamine). Neurotransmitters such as dopamine lower the threshold of neuronal cell firing in structures such as the insular/SII, SI cortices, the amygdala, and the VM cortex, so that the threshold for feeling and triggering somatic states is changed. Thus after a streak of few losses, the "thought" of another loss becomes more painful and triggers a stronger negative somatic state, whereas after a streak of few gains, the "thought" of another gain becomes more pleasurable and triggers a stronger positive somatic state.

While pre-existing negative somatic states reinforce subsequent negative states, they may impede the effectiveness of positive ones. Similarly, pre-existing positive states reinforce positive states, but they may impede negative ones. Thus in a losing market, the feeling is drawn towards pessimism. Decisions are more sensitized to the biasing influence of negative somatic states. In Overskeid's conceptualization, thinking that the market will reverse its negative course is a solution that contradicts the facts, so this creates a problem, i.e., a negative state that one wants to change. Therefore, under these conditions, the person is biased to accept the solutions that are least painful, such as cutting the losses short and rushing to sell. The opposite is the case during a growing market: the feeling is drawn to- 
wards optimism. Decisions become sensitized to the biasing influence of positive somatic states, and less sensitive to the biasing influence of negative somatic states.

Thus negative states breed pessimism and positive states breed optimism. In a state of pessimism it becomes more difficult to switch to optimism and vice versa. This influence is mediated by two separate mechanisms:

(1) At the level of the brain, i.e., before the next somatic state is triggered, pre-existing somatic states influence the threshold of neuronal cell firing in trigger structures (e.g., VM cortex), so that subsequent somatic states from thoughts (secondary inducers) are triggered more or less easily.

(2) At the level of the soma, i.e., after a somatic state has been triggered, pre-existing somatic states influence the feedback signals generated by newly triggered ones.

For example, suppose a negative somatic state is associated with high heart rate, while a positive somatic state is associated with low heart rate. In a negative state, i.e., when heart rate is high, subsequent negative somatic states are reinforced; keeping heart rate high is physiologically easier to achieve. On the other hand, positive somatic states may be impeded, i.e., switching from high to low heart rate is now more difficult. Both mechanisms bring convergent results: changing the magnitude of the somatic feedback signals that bias feelings and decisions.

It is important here to note the difference between a greater urge (i.e., appetite) to gain after a streak of losses, and the weaker somatic signals for biasing decisions. For example, when a person is hungry, there is a greater appetite for food. However, this appetite is actually generated from the state of hunger itself; "thoughts" of food exacerbate the hunger state and do not alleviate it. In other words, during hunger, decisions are desensitized to the biasing influence of positive somatic states. Promises of delicious food and great restaurants do not bias a hungry person towards waiting. Thus increased appetite for food during hunger reflects a stronger drive to escape the current state of hunger, or the conditions that created the aversive or negative state; the behavior is more energized by the aversive somatic states of hunger, as opposed to the positive somatic states of various delicious food options. By analogy, in a crashing market, a greater appetite for gain may develop. However, this increased appetite for gain reflects a stronger drive to escape the current state of loss. Decisions are more influenced by the aversive somatic states of losses, as opposed to the positive somatic states of possible good stock options. In other words, the investor is more biased towards stopping the ongoing loss (i.e., is more likely to sell and escape the current state), as opposed to choosing promising stock options.

\subsection{3. "Background" somatic states may differentially influence primary and secondary induction}

Although feedback signals from "background" somatic states modulate activity in neural structures concerned with primary (e.g., the amygdala) and secondary (e.g., the VM cortex) induction, their influence on the two processes can be different, i.e., background somatic states may sensitize primary induction, but desensitize secondary induction, or vice versa. Evidence suggests that the brain responds violently when an expected event fails to materialize. For example the striatum and anterior cingulate respond to patterns that either 
repeat or alternate (Huettel et al., 2002; Zweig, 2002). However, if a repeating pattern was broken, then increased activity occurs in areas of the striatum. The longer a pattern has previously repeated, the more the striatum responds when the pattern is broken (Huettel et al., 2002; Zweig, 2002). The work of Wolfram Schultz and colleagues (Schultz et al., 2000) presents similar evidence. Dopamine has long been recognized as playing a primary role in reward. Using cellular physiology and recording from dopamine neurons in the brain, Schultz and colleagues showed increased dopamine firing when monkeys are exposed to reward initially, but then the firing transfers to the cues predictive of the reward. However, Schultz and colleagues also showed that if a monkey was expecting a reward and it did not arrive, the cue that predicted the reward leads to dopamine release. However, the dopamine release will stop instantly if the reward failed to arrive as it was expected (Schultz et al., 2000).

Together, these studies lend support to the notion that "background" somatic states bias one's expectations, so that "thoughts" (i.e., secondary inducers) about an unexpected event (e.g., a possible loss after a streak of gains) become less effective, but actual occurrence of unexpected events, i.e., a real loss after a streak of gains (primary inducer) may sensitize the somatic marker circuitry in an opposite direction. This sensitization of primary and secondary induction in different directions, and the very quick, almost instantaneous, switch from one somatic state to another when an unexpected event occurs, can exert a disproportional impact on somatic state activation. Thus people's decision may get reversed completely if a disappointment was encountered, i.e., people may overreact, panic, and rush to sell if after a streak of several gains, another gain was expected and it did not materialize (Zweig, 2002). Similarly, if the market expectations were too low and an unexpected good performance was encountered, this may put investors into a state of "euphoria" and a possible rush to buy.

\subsection{Theoretical model}

Market economy favors conditions where investors take risks. There is probably an evolutionary advantage to taking risks. The work of Wolfram Schultz and his colleagues (Schultz et al., 2000) provides a good biological evidence for the reason why we take risks. If an animal received an unexpected (or unlikely) reward, the activity of dopamine neurons increases. Perhaps the greater release of dopamine after an unexpected reward is what prompts the taking of a risk. Without it, the organism may not explore new resources for food and consequently starve to death. By analogy, "perhaps without the dopamine signal to take a risk, modern investors would probably keep all their money under the mattresses" (Zweig, 2002). This suggests that risk taking in economic investment is modulated by somatic states. Background somatic states related to previous market news (e.g., a market loss) exert an influential role on the operation of the somatic marker circuitry and subsequent decisions and risk taking behaviors. There are several conditions under which somatic states that guide decisions can be altered by somatic states (emotions and moods) in the background (Fig. 10). 


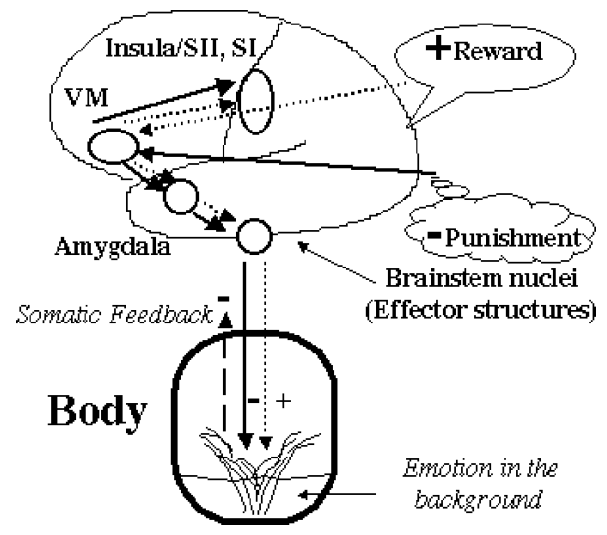

(A)

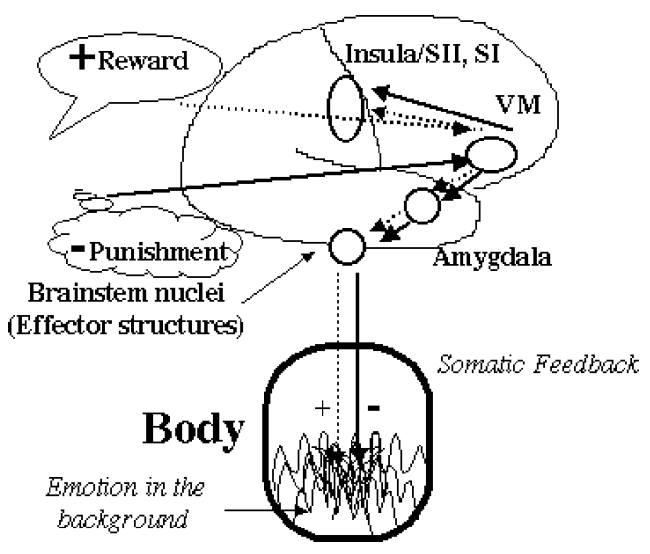

(B)

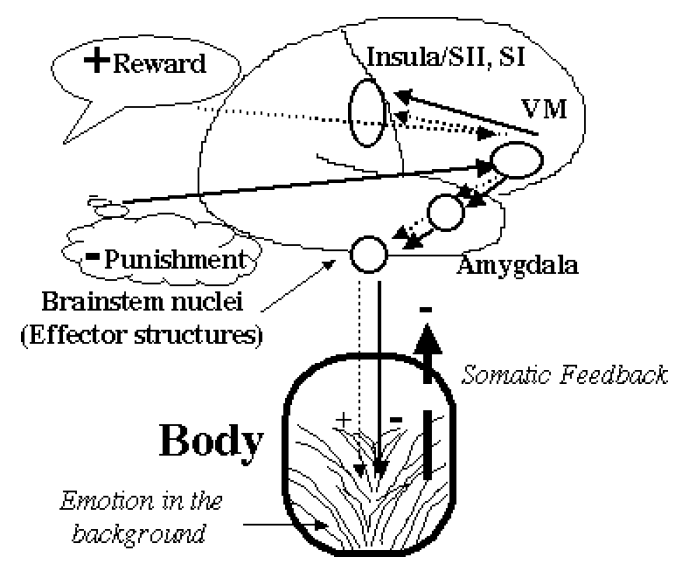

(C)

Fig. 10. (A) A schematic model of somatic state activation and decision-making in a hypothetical condition in which (1) the somatic state of the thought of punishment is greater than that of reward, and (2) the background emotion is neutral or weak. (B, C) A schematic model of somatic state activation and decision-making in a hypothetical condition in which (1) the somatic state of the thought of punishment is greater than that of reward, and (2) the background emotion is strong and incongruous with the triggered somatic states (B) or congruous with the triggered somatic states (C). Notes. (A) Background emotion is weak. Somatic states from thoughts of punishment (solid line) are stronger than those from reward (dotted line). When $(+)$ and (-) somatic states impinge on the emotional state in the background, the signal to noise ratio is high (big splash). The result is a strong somatic feedback to the brain. (B) Background emotion is strong and incongruous. Somatic states from thoughts of punishment (solid line) and from reward (dotted line) are the same as in (A). When (+) and (-) somatic states impinge on the emotional state in the background, the signal to noise ratio is low (no splash). (C) Background emotion is strong and congruous. Somatic states from thoughts of punishment (solid line) and from reward (dotted line) are the same as in (A). When $(+)$ and $(-)$ somatic states impinge on the emotional state in the background, the signal is in sink with the noise (i.e., it creates a strong splash). The result is an exaggerated somatic feedback to the brain. 


\subsubsection{Neutral or weak "background" somatic states}

When an almost neutral or weak somatic state exists in the background (e.g., when the market conditions are stable and uneventful), the triggering of somatic states from pondering a decision are more effective in overriding pre-existing somatic states and inducing physiological changes on their own. In this case, the signal (triggered somatic state) to noise (background state) ratio is high, and somatic states triggered from pondering decisions provide feedback signals to the brain, which bias behavior in the advantageous direction. Under these conditions, human decisions and risk taking behaviors are most sensitive to long term consequences, i.e., the individual's decisions are biased in favor of options that lead to the highest benefit in the long term future, and against options that lead to losses.

\subsubsection{Strong "background" somatic states}

When a strong somatic state exists in the background, somatic states triggered from secondary inducers (i.e., thoughts) are strongly altered: somatic states that are incongruous with "background" somatic states become weaker, and somatic states that are congruous become stronger. In the former case, the signal to noise ratio is too low, i.e., feedback signals from triggered somatic states (secondary inducers) are cancelled by the noise signals in the background. In the latter, the signal is in sink with the noise, so that the triggered somatic signals are amplified. Thus, in the event of a crashing market, "thoughts" that signal another loss become dominant and gain control over behavior, while "thoughts" that signal potential gain in the future become less effective. In contrast, in a growing market, "thoughts" that signal another gain win, while "thoughts" that signal potential market crash in the future become less effective in guiding decisions.

The same model also predicts that risk-taking behavior is modulated by the nature of somatic states in the background. We have suggested that "sure" rewards and punishments are processed via more posterior regions of the VM cortex, while "less probable" rewards and punishments are processed via more anterior regions. Since somatic states triggered from more posterior VM regions are stronger than those triggered from more anterior regions, it follows that there is a disproportionate increase in the strength of somatic states processed via the posterior, relative to the more anterior, VM cortices. Thus in accordance with the Prospect theory model, our Somatic marker model predicts that risk seeking in the face of sure loss is enhanced when the background state is negative. In other words, after a streak of several losses, there is a disproportionate increase in the aversion to another "sure" loss, so that seeking risky alternatives is increased. On the other hand, risk aversion in the face of sure gain is enhanced when the background state is positive. For example, after a streak of several gains, there is a disproportionate increase in the desire for another "sure" gain, so that seeking risky alternatives is decreased.

\subsubsection{Testing the model}

Testing Somatic marker model offers several predictions of human economic choice, which testing is only in its preliminary stage, and studies are currently conducted in order to test various predictions from this model. However, preliminary empirical support for the proposed model comes from two lines of studies: 
George Loewenstein and his colleagues (Loewenstein et al., 2001) have argued that decision-making is influenced by hot/cold states, i.e., in the "hot" state, decisions are driven by affect, and in the "cold" state, decisions are driven by cognition. They showed that the decision on how much pain medicine or food, for example, one needs to buy depends on whether the individual is in a "hot" state (i.e., in a state of pain or hunger) or a "cold" state (i.e., no pain or hunger). When the individual is in pain or hungry, the individual overestimates the amount of pain medicine or food, respectively, that one needs to buy. These findings are in line with our proposed model that when a strong somatic state of pain, for example, exists in the background, the triggering of a somatic state from pondering a decision that relates to pain, a state that is congruous with the pain state in the background, is exaggerated. Thus, similar to someone who is hungry and thinks of food, and the "thought" of food exacerbates the state of hunger, the "thought" of pain medicine in someone who is in pain exaggerates the somatic signal of how much pain the individual will feel in the future. Therefore, the final decision is biased towards over-buying pain medicine. This is analogous to the situation in which a person is too hungry and goes to a grocery store. The decision of how much food to buy in order to avoid hunger is altered by the hunger state itself, so that the person is likely to over-estimate the amount of food needed.

A complementary line of support comes from preliminary studies in neurological patients with lesions that preclude their normal capacity to react emotionally and trigger emotions (somatic states) in reaction to loss. At least in circumstances where normal individuals drop out of playing an investment task because of a heightened anxiety brought about by a streak of several losses, the poor somatic reaction of neurological patients to these losses enable them to continue investing, and thus outperform normal individuals (Shiv, Loewenstein, Bechara, Damasio, and Damasio; unpublished observations).

\section{Conclusion}

Emotions are a major factor in the interaction between environmental conditions and human decision processes, with these emotional systems (underlying somatic state activation) providing valuable implicit or explicit knowledge for making fast and advantageous decisions. Thus the somatic marker view of decision-making is anchored in the emotional side of humans as opposed to the construct of homo economicus. Although the view of maximizing utility of decision-making is pervasive and has a useful benchmark function, human decision-makers seldom conform to it. The process of deciding advantageously is not just logical but also emotional.

\section{Acknowledgments}

Supported by NIH Program Project Grant PO1 NS19632, NIDA RO1 DA11779-02, and DA12487-03. 


\section{References}

Abramson, L.Y., Seligman, M.E., Teasdale, J.D., 1978. Learned hopelessness in humans: critique and reformulation. J. Abnormal Psychology 87, 102-109.

Ackerly, S.S., Benton, A.L., 1948. Report of a case of bilateral frontal lobe defect. Proc. Assoc. Res. Nervous Mental Disease (Baltimore) 27, 479-504.

Anderson, S.W., Damasio, H., Jones, R.D., Tranel, D., 1991. Wisconsin card sorting test performance as a measure of frontal lobe damage. J. Clinical Exper. Neuropsychology 3, 909-922.

Anderson, S.W., Bechara, A., Damasio, H., Tranel, D., Damasio, A.R., 1999. Impairment of social and moral behavior related to early damage in the human prefrontal cortex. Nature Neurosci. 2 (11), 1032-1037.

Bechara, A., 2002. The role of emotion in judgment and decision-making: Evidence from neurological patients with orbitofrontal damage. Brain Cognition, in press.

Bechara, A., 2003. Risky business: Emotion, decision-making and addiction. J. Gambling Stud. 19 (1), $23-51$.

Bechara, A., Tranel, D., Damasio, H., Damasio, A.R., 1996. Failure to respond autonomically to anticipated future outcomes following damage to prefrontal cortex. Cerebral Cortex 6, 215-225.

Bechara, A., Damasio, H., Tranel, D., Damasio, A.R., 1997. Deciding advantageously before knowing the advantageous strategy. Science 275, 1293-1295.

Bechara, A., Damasio, H., Tranel, D., Anderson, S.W., 1998. Dissociation of working memory from decision making within the human prefrontal cortex. J. Neurosci. 18, 428-437.

Bechara, A., Damasio, H., Damasio, A.R., Lee, G.P., 1999. Different contributions of the human amygdala and ventromedial prefrontal cortex to decision-making. J. Neurosci. 19 (13), 5473-5481.

Bechara, A., Damasio, H., Damasio, A.R., 2000a. Emotion, decision-making, and the orbitofrontal cortex. Cerebral Cortex 10 (3), 295-307.

Bechara, A., Tranel, D., Damasio, H., 2000b. Characterization of the decision-making impairment of patients with bilateral lesions of the ventromedial prefrontal cortex. Brain 123, 2189-2202.

Bechara, A., Damasio, H., Damasio, A.R., 2001. Manipulation of dopamine and serotonin causes different effects on covert and overt decision-making. Soc. Neurosci. Abstracts 27.

Bechara, A., Tranel, D., Damasio, A.R., 2002. The somatic marker hypothesis and decision-making. In: Boller, F., Grafman, J. (Eds.), Handbook of Neuropsychology: Frontal Lobes. Elsevier, Amsterdam, pp. 117-143.

Bechara, A., Damasio, H., Damasio, A.R., 2003. The role of the amygdala in decision-making. In: ShinnickGallagher, P., Pitkanen, A., Shekhar, A., Cahill, L. (Eds.), The Amygdala in Brain Function: Basic and Clinical Approaches. Ann. New York Academy Sci., 356-369.

Berkowitz, L., 1993. Towards a general theory of anger and emotional aggression: Implications of the cognitiveneoassociationistic perspective for the analysis of anger and other emotions. In: Wyer, R.S., Srull, T.K. (Eds.), Advances in Social Cognition. Lawrence Earlbaum Associates, Hillsdale, NJ, pp. 1-46.

Berns, G.S., McClure, S.M., Pagnoni, G., Montague, P.R., 2001. Predictability modulates human brain response to reward. J. Neurosci. 21 (8), 2703-2708.

Bickel, W.K., Degrandpre, R.J., Higgins, S.T., 1995. The behavioral economics of concurrent drug reinforcers. A review and reanalysis of drug self-administration research. Psychopharmacology 118 (3), 250-259.

Blessing, W.W., 1997. Anatomy of the lower brainstem. In: The Lower Brainstem and Bodily Homeostasis. Oxford Univ. Press, New York, Oxford, pp. 29-99.

Brickner, R.M., 1932. An interpretation of frontal lobe function based upon the study of a case of partial bilateral frontal Lobectomy. Localization of function in the cerebral cortex. Proc. Assoc. Res. Nervous Mental Disease (Baltimore) 13, 259-351.

Buchel, C., Morris, J., Dolan, R.J., Friston, K.J., 1998. Brain systems mediating aversive conditioning: An eventrelated fmri study. Neuron 20 (5), 947-957.

Cacioppo, J.T., Berntson, G.G., Larsen, J.T., Poehlmann, K.M., Ito, T.A., 2000. The psychophysiology of emotion. In: Lewis, R., Haviland-Jones, J. (Eds.), The Handbook of Emotion. Guiford Press, New York, pp. 173191.

Damasio, A.R., 1994. Descartes' Error: Emotion, Reason, and the Human Brain. Grosset/Putnam, New York.

Damasio, A.R., 1995. Toward a neurobiology of emotion and feeling: operational concepts and hypotheses. Neurosci. $1,19-25$.

Damasio, A.R., 1999. The Feeling of What Happens: Body and Emotion in the Making of Consciousness. Harcourt Brace \& Co., New York. 
Damasio, A.R., 2003. Looking for Spinoza: Joy, Sorrow, and the Feeling Brain. Harcourt Brace \& Co., New York.

Damasio, A.R., Tranel, D., Damasio, H., 1990. Individuals with sociopathic behavior caused by frontal damage fail to respond autonomically to social stimuli. Behavi. Brain Res. 41, 81-94.

Damasio, A.R., Tranel, D., Damasio, H., 1991. Somatic markers and the guidance of behavior: Theory and preliminary testing. In: Levin, H.S., Eisenberg, H.M., Benton, A.L. (Eds.), Frontal Lobe Function and Dysfunction. Oxford Univ. Press, New York, pp. 217-229.

Davidson, R.J., Irwin, W., 1999. The functional neuroanatomy of emotion and affective style. Trends Cog. Sci. 3 (1), 11-21.

Deglin, V.L., Kinsbourne, M., 1996. Divergent thinking styles of the hemispheres: How syllogisms are solved during transitory hemisphere suppression. Brain Cog. 31, 285-307.

Dolan, R.J., Fletcher, P., Morris, J., Kapur, N., Deakin, J.F.W., Frith, C.D., 1996. Neural activation during covert processing of positive emotional facial expressions. Neuroimage 4 (3), 194-200.

Einhorn, H.J., Hogarth, R.M., 1985. Ambiguity and uncertainty in probabilistic inference. Psychological Rev. 92, 433-461.

Ellsberg, D., 1961. Risk, ambiguity, and the savage axioms. Quart. J. Econ. 75, 643-669.

Eslinger, P.J., Damasio, A.R., 1985. Severe disturbance of higher cognition after bilateral frontal lobe ablation: Patient evr. Neurology 35, 1731-1741.

First, M.B., Spitzer, R.L., Gibbon, M., Williams, J.B.W., 1997. Structured Clinical Interview for Dsm-Ivaxis I Disorders, Research Version, Non-Patient Edition (Scid-I/Np). Biometrics Research Department, New York State Psychiatric Institute, New York.

Frith, C.D., Friston, K., Liddle, P.F., Frackowiak, R.S.J., 1991. Willed action and the prefrontal cortex in man. A study with pet. Proc. Roy. Soc. London Ser. B Biol. Sci. 244 (1311), 241-246.

Fuster, J.M., 1996. The Prefrontal Cortex. Anatomy, Physiology, and Neuropsychology of the Frontal Lobe. Raven Press, New York.

Gallagher, M., McMahan, R.W., Schoenbaum, G., 1999. Orbitofrontal cortex and representation of incentive value in associative learning. J. Neurosci. 19 (15), 6610-6614.

Gray, J.R., 1999. A bias toward short-term thinking in threat-related negative emotional states. Personality Soc. Psychology Bull. 25 (1), 65-75.

Green, L., Fry, A., Myerson, J., 1994a. Discounting of delayed rewards: A life-span comparison. Psycholog. Sci. 5, 33-36.

Green, L., Fristoe, N., Myerson, J., 1994b. Temporal discounting and preference reversals in choice between delayed outcomes. Psychonomic Bull. Rev. 1 (3), 383-389.

Greene, J.D., Sommerville, R.B., Nystrom, L.E., Darley, J.M., Cohen, J.D., 2001. An fmri investigation of emotional engagement in moral judgment. Science 293 (5537), 2105-2108.

Herrnstein, R.J., Prelec, D., 1991. Melioration. A theory of distributed choice. J. Econ. Perspect. 5 (3), $137-156$.

Huettel, S.A., Mack, P.B., McCarthy, G., 2002. Perceiving patterns in random series: Dynamic processing of sequence in prefrontal cortex. Nature Neurosci. 5 (5), 485-490.

Janis, I.L., Mann, L., 1977. Decision-Making: A Psychological Analysis of Conflict, Choice, and Commitment. Free Press, New York.

Kirby, K.N., Herrnstein, R.J., 1995. Preference reversals due to myopic discounting of delayed reward. Psycholog. Sci. 6 (2), 83-89.

Knowlton, B.J., Mangels, J.A., Squire, L.R., 1996. A neostriatal habit learning system in humans. Science 273 , 1399-1402.

LaBar, K.S., Gatenby, J.C., Gore, J.C., LeDoux, J.E., Phelps, E.A., 1998. Human amygdala activation during conditioned fear acquisition and extinction: A mixed-trial fmri study. Neuron 20, 937-945.

LeDoux, J., 1996. The Emotional Brain: The Mysterious Underpinnings of Emotional Life. Simon and Schuster, New York.

Loewenstein, G.F., Weber, E.U., Hsee, C.K., Welch, N., 2001. Risk as feelings. Psycholog. Bull. 127 (2), $267-$ 286.

Manes, F., Sahakian, B., Clark, L., Rogers, R., Antoun, N., Aitken, M., Robbins, T., 2002. Decision-making processes following damage to the prefrontal cortex. Brain 125, 624-639.

Mann, L., 1992. Stress, affect, and risk taking. In: Frank, Y.J. (Ed.), Risk-Taking Behavior. Wiley, Chichester, England, pp. 202-230. 
Milner, B., Petrides, M., Smith, M.L., 1985. Frontal lobes and the temporal organization of memory. Human Neurobiology 4, 137-142.

Milner, B., Corsi, P., Leonard, G., 1991. Frontal lobe contribution to recency judgments. Neuropsychologia 29, 601-618.

Mogenson, G.J., 1987. Limbic motor integration. In: Epstein, A., Morrison, A.R. (Eds.), Progress in Psychobiology and Physiological Psychology. Academic Press, New York, pp. 117-170.

Monterosso, J., Ehrman, R., Napier, K., O’Brien, C.P., Childress, A.R., 2001. Three decision-making tasks in cocaine-dependent patients: Do they measure the same construct? Addiction 96 (12), 1825-1837.

Morris, J.S., Ohman, A., Dolan, R.J., 1999. A subcortical pathway to the right amygdala mediating "unseen" fear. Proc. Nat. Acad. Sci. USA 96 (4), 1680-1685.

Nauta, W.J.H., 1971. The problem of the frontal lobes: A reinterpretation. J. Psychiat. Res. 8, 167-187.

Nichelli, P., 2002. The processing of temporal information in the frontal lobe. In: Grafman, J. (Ed.), Handbook of Neuropsychology: Frontal Lobes. Elsevier, Amsterdam, pp. 175-193.

Ongur, D., Price, J.L., 2000. The organization of networks within the orbital and medial prefrontal cortex of rats, monkeys and humans. Cerebral Cortex 10 (3), 206-219.

Overskeid, G., 2000. The slave of passions: Experiencing problems and selecting solutions. Rev. General Psychology 4 (3), 284-309.

Pagnoni, G., Zink, C.F., Montague, P.R., Berns, G.S., 2002. Activity in human ventral striatum locked to errors of reward prediction. Nature Neurosci.

Pardo, J.V., Pardo, P.J., Janer, K.W., Raichle, M.E., 1990. The anterior cingulate cortex mediates processing selection in the stroop attentional conflict paradigm. Proc. Nat. Acad. Sci. USA 87 (1), 256-259.

Petit, L., Orssaud, C., Tzourio, N., Salamon, G., Mazoyer, B., Berthoz, A., 1993. Pet study of voluntary saccadic eye-movements in humans. Basal ganglia. Thalamocortical system and cingulate cortex involvement. J. Neurophysiology 69 (4), 1009-1016.

Petrides, M., 1985. Deficits on conditional associative learning tasks after frontal and temporal lobe lesions in man. Neuropsychologia 23, 601-614.

Petrides, M., Alivisatos, B., Evans, A.C., Meyer, E., 1993. Dissociation of human mid-dorsolateral from posterior dorsolateral frontal cortex in memory processing. Proc. Nat. Acad. Sci. USA 90, 873-877.

Posner, M.I., Petersen, S.E., 1990. The attention system of the human brain. Annual Rev. Neurosci. 13, $25-42$.

Posner, M.I., Petersen, S.E., Fox, P.T., Raichle, M.E., 1988. Localization of cognitive operations in the human brain. Science 240 (4859), 1627-1631.

Rogers, R.D., Everitt, B.J., Baldacchino, A., Blackshaw, A.J., Swainson, R., Wynne, K., Baker, N.B., Hunter, J., Carthy, T., Booker, E., et al., 1999a. Dissociable deficits in the decision-making cognition of chronic amphetamine abusers, opiate abusers, patients with focal damage to prefrontal cortex, and tryptophan-depleted normal volunteers: Evidence for monoaminergic mechanisms. Neuropsychopharmacology 20 (4), 322-339.

Rogers, R.D., Owen, A.M., Middleton, H.C., Williams, E.J., Pickard, J.D., Sahakian, B.J., Robbins, T.W., 1999 b. Choosing between small, likely rewards and large, unlikely rewards activates inferior and orbital prefrontal cortex. J. Neurosci. 20, 9029-9038.

Roozendaal, B., Cahill, L., McGaugh, J.L., 1996. Interaction of emotionally activated neuromodulatory systems in regulating memory storage. In: Ishikawa, K., McGaugh, J.L., Sakata, H. (Eds.), Brain Processes and Memory. Elsevier, Amsterdam, pp. 39-54.

Saver, J.L., Damasio, A.R., 1991. Preserved access and processing of social knowledge in a patient with acquired sociopathy due to ventromedial frontal damage. Neuropsychologia 29, 1241-1249.

Schneider, W., Shiffrin, R.M., 1977. Controlled and automatic human information processing. Psycholog. Rev. 84, 1-66.

Schoenbaum, G., Chiba, A.A., Gallagher, M., 1998. Orbitofrontal cortex and basolateral amygdala encode expected outcomes during learning. Nature Neurosci. 1 (2), 155-159.

Schultz, W., Tremblay, L., Hollerman, J.R., 2000. Reward processing in primate orbitofrontal cortex and basal ganglia. Cerebral Cortex 10 (3), 272-283.

Schwartz, N., Clore, G.L., 1983. Mood, misattribution, and judgements of well-being: Information and directive functions of affective states. J. Personality Soc. Psychology 45, 513-523.

Semendeferi, K., Armstrong, E., Schleicher, A., Zilles, K., Van Hoesen, G.W., 2001. Prefrontal cortex in humans and apes: A comparative study of area 10. Amer. J. Physical Anthropology 114 (3), 224-241. 
Semendeferi, K., Lu, A., Schenker, N., Damasio, H., 2002. Humans and great apes share a large frontal cortex. Nature Neurosci. 5 (3), 272-276.

Shimamura, A.P., Janowsky, J.S., Squire, L.R., 1990. Memory for the temporal order of events in patients with frontal lobe lesions and amnesic patients. Neuropsychologia 28, 803-813.

Smith, M.L., Milner, B., 1984. Differential effects of frontal lobe lesions on cognitive estimation and spatial memory. Neuropsychologia 22, 697-705.

Tranel, D., Bechara, A., Damasio, H., Damasio, A.R., 1996. Fear conditioning after ventromedial frontal lobe damage in humans. Soc. Neurosci. Abstracts 22, 1108.

Tranel, D., Bechara, A., Denburg, N.L., 2002. Asymmetric functional roles of right and left ventromedial prefrontal cortices in social conduct, decision-making, and emotional processing. Cortex 38, 589-612.

Tranel, D., Damasio, A.R., 1993. The covert learning of affective valence does not require structures in hippocampal system or amygdala. J. Cog. Neurosci. 5, 79-88.

Tversky, A., Kahneman, D., 1981. The framing of decisions and the rationality of choice. Science 211, $453-458$.

Tversky, A., Kahneman, D., 1974. Judgment under uncertainty: Heuristics and biases. Science 185, 1124-1131.

von Neumann, J., Morgenstern, O., 1944. Theory of Games and Economic Behavior. Princeton Univ. Press, Princeton, NJ.

Welt, L., 1888. Uber Charaktervaranderungen Des Menschen Infoldge Von Lasionen Des Stirnhirns. Dtsch. Arch. Klin. Med. 42, 339-390.

Wolford, G., Miller, M.B., Gazzaniga, M., 2000. The left hemisphere's role in hypothesis formation. J. Neurosci. 20, 1-4, RC64.

Zajonc, R.B., 1984. On the primacy of affect. Amer. Psychologist 39, 117-123.

Zweig, J., 2002. Are you wired for wealth? Money, 75-83. 